\title{
BLENDERS IN CENTER UNSTABLE HÉNON-LIKE FAMILIES: WITH AN APPLICATION TO HETERODIMENSIONAL BIFURCATIONS
}

\author{
LORENZO J. DÍAZ, SHIN KIRIKI, AND KATSUTOSHI SHINOHARA
}

\begin{abstract}
We give an explicit family of polynomial maps called center unstable Hénon-like maps and prove that they exhibits blenders for some parametervalues. Using this family, we also prove the occurrence of blenders near certain non-transverse heterodimensional cycles under high regularity assumptions. The proof involves a renormalization scheme along heteroclinic orbits. We also investigate the connection between the blender and the original heterodimensional cycle.
\end{abstract}

\section{INTRODUCTION}

This paper has two main goals. The first one is to exhibit an explicit family of quadratic polynomial maps in dimension three (center unstable Hénon-like families) with blenders. The second one is to prove the occurrence of blenders near certain non-transverse heterodimensional cycles and the connections between them under high regularity assumptions. The two previous results are related as follows: associated to the non-transverse heterodimensional cycles there are renormalization schemes converging to center unstable Hénon-like families.

We now briefly discuss the three main topics of this paper: blenders, nontransverse heterodimensional cycles, and renormalization. In what follows we assume that the dimension of the ambient space is three.

Blenders and Hénon-like families. Blenders (see Definition 2.1) only appear in dimension greater than or equal to 3 and are just a special type of hyperbolic sets $\Lambda$ of diffeomorphisms $f$ which are maximal invariant in a neighborhood $\Delta$, that is, $\Lambda=\cap_{n \in \mathbb{Z}} f^{n}(\Delta)$. We consider the case where the stable direction is onedimensional. Then the blender $\Lambda$ has a dominated splitting with three hyperbolic directions (the stable, the center unstable, and the strong unstable directions). A key property of a blender is its internal dynamical configuration that implies that every curve which crosses a distinctive open region of $\Delta$ and is almost tangent to the one-dimensional strong unstable direction intersects the local stable manifold of $\Lambda$. This roughly means that $\Lambda$ topologically behaves as a hyperbolic set with stable manifold of dimension two. Another relevant property of a blender is its robustness: for diffeomorphisms $g$ near $f$ the continuation of the hyperbolic set $\Lambda$ for $g$ is also a blender.

A blender is an important ingredient for obtaining robust non-hyperbolic dynamics: blenders play a similar role as the thick horseshoes introduced by Newhouse

Date: March 17, 2022.

2000 Mathematics Subject Classification. Primary: 37C20; 37C29; 37C70; Secondary: 37C25.

Key words and phrases. blender, Hénon-like family, heterodimensional cycle, renormalization. 
[14, 15. They are used to turn heterodimensional cycles and homoclinic tangencies $C^{1}$-robust, see 4,5 . We remark that recently some authors have introduced blenders whose center unstable direction is indecomposable and has dimension $\geq 2$, see [13, 1].

We study how blenders occur. As far as we know, their construction involves series of perturbations which are genuinely $C^{1}$, see for instance [4, 5]. We present an explicit family of quadratic maps (which we call a center unstable Hénon-like family) with blenders. A novelty here is that the blenders are obtained without perturbations and their occurrence only involves an appropriate selection of parameters of the family.

Theorem 1.1. Consider the center unstable Hénon-like family

$$
G_{\xi, \mu, \kappa, \eta}(x, y, z)=\left(\xi x+y, \mu+y^{2}+\kappa x^{2}+\eta x y, y\right), \quad \xi>1 .
$$

There is an open set $\mathcal{B}$ of parameters $(\xi, \mu, \kappa, \eta)$ such that any diffeomorphism $F$ $C^{r}$-close to $G_{\xi, \mu, \kappa, \eta}$ with $(\xi, \mu, \kappa, \eta) \in \mathcal{B}$ has a blender.

One important feature of the family $(1.1)$ is the existence of the term $y$ in the $x$ component. This term gives us a superposition property (see Definition 2.1) of the hyperbolic set which enables us to obtain the blender, compare the family treated in $[9$.

Coexistence of critical and noncritical dynamics. Homoclinic tangencies and Hénon-like dynamics are in the core of the so-called critical dynamics, while heterodimensional cycles are genuine bifurcations of non-critical dynamics, for more details see Preface of [6]. There are many cases where the effects of the critical and the non-critical dynamics overlap: the system has a critical region and a noncritical one and there are transitions between these two regions. We study such kind of configuration in dimension three: diffeomorphisms having two saddles of different indices (dimension of the unstable direction) whose invariant manifolds are cyclically related by a heterodimensional cycle with non-transverse heteroclinic intersections. This configuration (depicted in Figure 1.1) is called a non-transverse heterodimensional cycle: the two invariant manifolds of dimension one meet quasitransversally and the two-dimensional ones have a tangential intersection. The dynamics close to the saddles and the quasi-transverse heteroclinic intersection provide the non-critical part of the dynamics, while the critical one is given by the heteroclinic tangency.

The non-transverse cycle that we consider contains a heterodimensional tangency. In the $C^{1}$ case, it is known that the unfolding of these tangencies leads to robustly non-dominated dynamics and in some cases to very intermingled dynamics related to universal dynamics introduced in [3], see [8] for detail. In the $C^{2}$ case, the non-transverse cycles are not special in the following sense: there exists $C^{2}$ open setw of $n$-dimensional $(n>2)$ diffeomorphisms having heterodimensional tangencies in a coindex- $(n-2)$ heterodimensional cycle, see [12. Note that the occurence of $C^{2}$ Newhouse phenomenon and strange attractors were observed in 11.

Using Theorem 1.1 and the renormalization scheme that we will present below, we prove the existence of blenders near some of these non-transverse heterodimensional cycles under high regularity assumptions, see Corollary 1.3. Let us observe that by adjusting the parameters, we can obtain convergence of the renormalization 


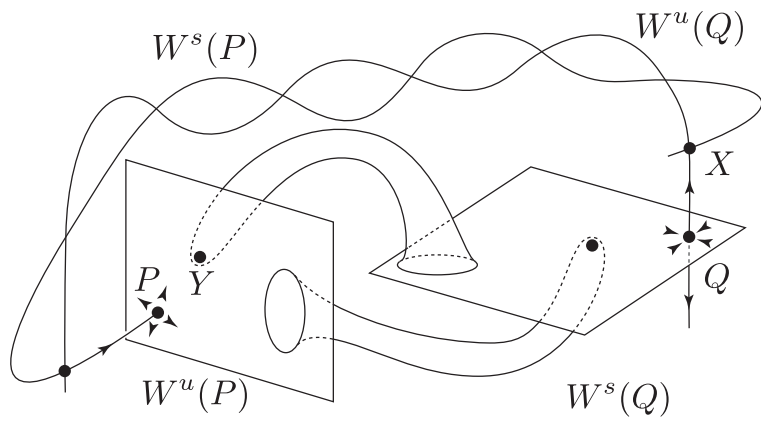

(a)

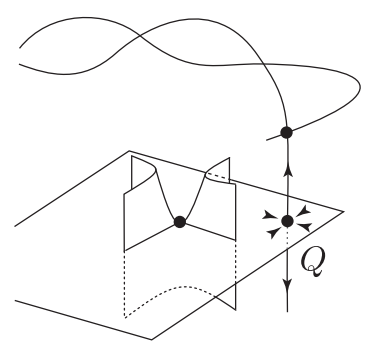

(b)

FiguRE 1.1. Non-transverse heterodimensional cycles

to different types of Hénon-like maps, see the comment after Theorem 1.2 This illustrates the richness of the dynamics near these cycles.

Renormalization. Renormalization means providing a sequence of local coordinate changes near a tangency point and reparametrizations, which gives a sequence of return maps along heteroclinic orbits converging to a limit map with interesting dynamics. Using a renormalization scheme one can translate relevant properties of the limit maps to some diffeomorphisms close to the one with the tangency.

Renormalization methods play an important role in the study of homoclinic bifurcations (dynamics at homoclinic tangencies). This method leads to the approximation of the dynamics by quadratic families and allows to translate properties of such families (such as existence of strange attractors and sinks, or thick hyperbolic sets) to properties of the diffeomorphisms, see for instance [16, Chapter 3].

So far, renormalizations have not been sufficiently exploited in the context of heterodimensional bifurcations. We consider renormalization schemes for nontransverse heterodimensional cycles. Depending on the conditions satisfied by the diffeomorphism, this renormalization may converge to different types of dynamics. We wonder if this limit map may exhibit blenders. In general, it is not always the case. We prove that, under some (degenerate) conditions, the renormalization converges to the center unstable Hénon-like families in Theorem 1.1, see Theorem 1.2 .

We now state precise definitions and results.

Definitions and statements of the results. Consider diffeomorphisms $f$ defined on a closed three-dimensional manifold $M$ having hyperbolic periodic points $P$ and $Q$ of saddle type with different indices (dimension of the unstable bundle and denoted by $\operatorname{index}(\cdot))$. We assume that $f$ has a non-transverse heterodimensional cycle associated to the saddles $P$ and $Q$ (assume that index $(P)=2>\operatorname{index}(Q)=$ 1). This means that

$$
W^{s}(P) \cap W^{u}(Q) \neq \emptyset \quad \text { and } \quad W^{s}(Q) \cap W^{u}(P) \neq \emptyset,
$$

where the first intersection between one-dimensional manifolds is quasi-transverse, that is, there is $X \in W^{s}(P) \cap W^{u}(Q)$ such that

$$
\operatorname{dim}\left(T_{X} W^{s}(P)+T_{X} W^{u}(Q)\right)=\operatorname{dim}\left(E^{s}(P)\right)+\operatorname{dim}\left(E^{u}(Q)\right),
$$

and the two-dimensional manifolds $W^{u}(P)$ and $W^{s}(Q)$ have a non-transverse intersection (a tangency) along the orbit of a point $Y$. This geometrical configuration is 
depicted in Figure 1.1 (the tangency in (a) is elliptic and the one in (b) is hyperbolic, see [11]).

Associated to the heteroclinic point $X$, there is a transition from a neighborhood $U_{Q}$ of $Q$ to a neighborhood $U_{P}$ of $P$ following the orbit of $X$. Similarly, associated to $Y$ there is a transition from $U_{P}$ to $U_{Q}$, see also Figure 3.1. We impose some conditions to these transitions in equation (3.3).

Let $\operatorname{per}(P)$ and $\operatorname{per}(Q)$ be the periods of $P$ and $Q$, respectively. Denote the eigenvalues of $D f^{\operatorname{per}(P)}(P)$ and $D f^{\operatorname{per}(Q)}(Q)$ by $\tilde{\lambda}, \tilde{\zeta}, \tilde{\sigma}$ and $\lambda, \zeta, \sigma$, respectively. We assume that

$$
|\tilde{\lambda}|<1<|\tilde{\sigma}|<|\tilde{\zeta}|, \quad|\lambda|<|\zeta|<1<|\sigma|
$$

and

$$
\left.|| \tilde{\sigma} \tilde{\zeta}\right|^{k} \sigma \zeta^{2}|,|\left|\tilde{\sigma}^{-3} \tilde{\zeta}\right|^{k} \sigma^{-1}|,||\tilde{\lambda} \tilde{\sigma}|^{k} \sigma \mid<1, \quad \text { where } k=\frac{\log |\lambda|^{-1}}{\log |\tilde{\zeta}|} .
$$

As we mentioned, bifurcations of non-transverse heterodimensional cycles exhibit a rich variety of dynamics. Depending on the way of unfolding, one may reach different types of dynamics. In the following theorem, starting from a sixparameter family of diffeomorphisms (where the parameters describe the position of the continuations of the heteroclinic points above), we select a two-parameter sub-family converging to a Hénon-like family: there is a renormalization scheme near the tangency providing a sequence of maps $\left\{F_{\mu_{k}(\bar{\mu}), \nu_{k}}\right\}$, reparametrizations $\mu_{k}$, and parameters $\nu_{k}$ such that the family converges to a Hénon-like family.

Theorem 1.2. Consider $f \in \operatorname{Diff}^{r}(M), \operatorname{dim}(M)=3$ and $r \geq 2$, with a nontransverse heterodimensional cycle associated with saddles $P$ and $Q$ with heteroclinic orbits $X$ (quasi-transverse point) and $Y$ (tangency point).

Assume that

- The local dynamics around $P$ and $Q$ are linearized as in (3.1),

- the eigenvalues of $D f^{\operatorname{per}(P)}(P)$ and $D f^{\operatorname{per}(Q)}(Q)$ satisfy (1.3) and (1.4),

- the transitions between $P$ and $Q$ satisfy (3.3), (3.6) and (3.7).

Then there is a six-parameter family $\left\{f_{\mu, \nu}\right\}_{\mu, \nu \in[-\epsilon, \epsilon]^{3}} \subset \operatorname{Diff}^{r}(M)$ with $f_{\mathbf{0}, \mathbf{0}}=f$, satisfying the following: for any real number $\xi>0$, there are

- a sequence of coordinate changes $\Psi_{k}: K \rightarrow M$ defined near the tangency point $Y$, where $K$ is a compact neighborhood of the origin of $\mathbb{R}^{3}$ that can be taken arbitrarily large,

- reparametrizations $\mu_{k}: I \rightarrow \mathbb{R}^{3}$, where $I$ is a compact neighborhood of 0 in $\mathbb{R}$ that can be taken arbitrarily large,

- parameter values $\nu_{k} \in \mathbb{R}^{3}$,

- a sequence of pairs of natural numbers $\left(m_{k}, n_{k}\right)_{k}, m_{k}, n_{k} \rightarrow \infty$, and natural numbers $N_{1}$ and $N_{2}$ independent of $k$ and $\xi$,

such that the map

$$
F_{\mu_{k}(\bar{\mu}), \nu_{k}}(\overline{\mathbf{x}})=\left(\left.\Psi_{k}^{-1}\right|_{\Psi_{k}(K)}\right) \circ f_{\mu_{k}(\bar{\mu}), \nu_{k}}^{N_{2}+m_{k}+N_{1}+n_{k}} \circ \Psi_{k}(\overline{\mathbf{x}})
$$

satisfies the following properties:

(1) Suppose $(\bar{\mu}, \overline{\mathbf{x}})$ is contained in a (fixed) compact set of $\mathbb{R} \times \mathbb{R}^{3}$, then the sequence $\left\{\left(\mu_{k}(\bar{\mu}), \nu_{k}\right)\right\}$ converges to $(\mathbf{0}, \mathbf{0}) \in \mathbb{R}^{6}$ and the sequence $\left\{\Psi_{k}(\overline{\mathbf{x}})\right\}$ converges to the tangency point $Y$ as $k \rightarrow+\infty$. 
(2) The sequence of maps $\left\{F_{\mu_{k}(\bar{\mu}), \nu_{k}}\right\}$ converges in the $C^{r}$ topology to a one-parameter family conjugate to

$$
G_{\mu}(x, y, z)=G_{\xi, \mu, \kappa_{1}, \kappa_{2}}(x, y, z)=\left(\xi x+y, \mu+y^{2}+\kappa_{1} x^{2}+\kappa_{2} x y, y\right),
$$

where $\kappa_{1}, \kappa_{2}$ are constants depending only on $f$.

An immediate consequence of this theorem and Theorem 1.1 is the following:

Corollary 1.3. Let $f$ be a $C^{r}$ diffeomorphisms $(r \geq 2)$ satisfying the hypotheses of Theorem 1.2. Then every $C^{r}$-neighborhood of $f$ contains an open set of diffeomorphisms having blenders.

Let us give a comment to Theorem 1.2 The numbers $\left(m_{k}, n_{k}\right)$ correspond to the consecutive times during which the points stay close to the saddles $P$ and $Q$, respectively. The selection of these numbers determines convergence of the renormalization and the number $\xi$. This choice may lead to $\xi>1$ (center unstable Hénon-like maps) or $\xi<1$ (center stable Hénon-like maps). This means that arbitrarily close to the original system there are both types of dynamics.

Connecting one-dimensional invariant manifolds. We now show an application of the methods above. To exploit completely the consequence of our techniques is beyond the goal of this paper (it is a part of on-going research project).

Theorem 1.2 asserts that arbitrarily $C^{r}$-close to certain types of diffeomorphisms $f$ having a non-transverse heterodimensional cycle, there exist diffeomorphisms $g$ having center unstable blenders $\Lambda_{g}$ near the point of tangency. The renormalization gives us some local information, but does not provide information about the connections between $\Lambda_{g}$ and the continuations $P_{g}, Q_{g}$ of $P, Q$ (semi-global information). Since the dynamics is not dominated close to the tangency point, it is not easy to describe the relative positions of the invariant manifolds of the these sets.

Under some additional hypotheses on the contracting multipliers of $Q$, the next theorem gives the creation of robust intersections between the "one-dimensional" invariant manifolds $W^{s}\left(\Lambda_{g}, g\right)$ and $W^{u}\left(Q_{g}, g\right)$. This intersection is in principle harder to obtain than the one between the "two-dimensional" invariant manifolds.

Theorem 1.4. Let $f$ be a diffeomorphism having a non-transverse heterodimensional cycle associated to saddles $P$ and $Q$ satisfying the hypothesis of Theorem 1.2. Consider a real number $\alpha>0$ satisfying $\alpha<\frac{\log |\lambda|}{\log |\zeta|}-1$, where $|\lambda|<|\zeta|$ are the moduli of contracting eigenvalues of $D f^{\operatorname{per}(Q)}(Q)$ in (1.3). Then, for every $r \geq 2$, arbitrarily $C^{1+\alpha}$ close to $f$, there exists a $C^{r}$ diffeomorphism $g$ having a centerunstable blender $\Lambda_{g}$ such that $W_{\mathrm{loc}}^{s}\left(\Lambda_{g}, g\right) \cap W^{u}\left(Q_{g}, g\right) \neq \emptyset$ holds $C^{1}$-robustly.

Note that the $C^{1}$-robustness in the conclusion of Theorem 1.4 implies the $C^{r}$ robustness of the connection for all $r>1$. The number $\alpha$ in the theorem is in $(0,1 / 2)$, see Lemma 3.1 in Section 3.3 .

In the $C^{1}$-topology, the connecting lemma of Hayashi [10] and the constructions in 4 tell us that there are diffeomorphisms arbitrarily $C^{1}$-close to $f$ with robust cycles. We wonder if this is possible to obtain such connections for systems $C^{r}$ close to $f$ for $r>1$. We use a detailed estimate on the hyperbolic behavior of the limit map obtained in the renormalization process to get robust intersections for $C^{1+\alpha}$-approximations. As $\alpha$ is in $(0,1 / 2)$, we do not obtain, for example, a $C^{2}$-result. 
The above result gives a connection from $Q_{g}$ to $\Lambda_{g}$. However, in general it is also difficult to get non-empty intersections between $W^{s}\left(Q_{g}, g\right)$ and $W^{u}\left(\Lambda_{g}, g\right)$. This difficulty is due to the fact that the transition map from $P$ to $Q$ does not preserves the "central direction". Thus, the question still remains whether or not a $C^{r}$-robust heterodimensional cycle can be created from a bifurcation of the heterodimensional tangency.

To get such intersections one can consider two possible directions. The first one is to get a renormalization scheme providing further geometrical information of the cycles. This strategy is well exploited in the renormalizations in [17, 18, where the dynamics is normally hyperbolic (which is not our case). Another direction is to consider heterodimensional tangencies as in 8 associated to robustly nonhyperbolic transitive sets. These sets have rich structures that may help to find such intersections.

Organization of the paper. In Section 2 we introduce the definition of a blender, state a result guaranteeing its existence, and prove Theorem 1.1 about the occurrence of blenders for some Hénon-like families. In Section 3 we describe the class of non-transverse heterodimensional cycles we consider. This description involves properties of the transitions between the saddles in the cycle following the nontransverse heteroclinic orbits and the local dynamics near the saddles. The sixparameter family of diffeomorphisms in Theorem 1.2 is presented in Section 4. In Section 5 we introduce the renormalization scheme and prove Theorem 1.2 . Finally, in Section 6. Theorem 1.4 is shown by previous theorems and a certain $C^{1+\alpha}$ perturbation which is performed locally near the local unstable manifold of the continuation of $Q$.

\section{Blenders for Center unstable HÉNON-LIKE MAPS}

In this section, we consider diffeomorphisms which are $C^{r}$-near the center unstable Hénon-like endomorphism

$$
G_{\mu, \kappa, \xi}(x, y, z)=\left(y, \mu+y^{2}+\kappa z^{2}, \xi z+y\right) .
$$

This map is conjugate to the Hénon family in 1.1 when $\eta=0$ by the coordinate change

$$
\tilde{\Theta}:(x, y, z) \longmapsto(z, y, x) .
$$

We will prove the existence of blenders for diffeomorphisms close to $G_{\mu, \kappa, \xi}$ where the parameters are in some specific ranges (see Theorem 1.1).

2.1. Conditions for the existence of blenders. Before going to the proof of the theorem, let us recall the definition of a blender and sufficient conditions for the existence of blenders in [2, §1]. First, we give an axiomatic definition of a blender.

Definition 2.1 (Blender, Definition 3.1 in [5]). Let $f: M \rightarrow M$ be a diffeomorphism. A transitive hyperbolic compact set $\Lambda$ of $f$ with index $k, k \geq 2$, is a cu-blender if there are a $C^{1}$-neighborhood $\mathcal{U}$ of $f$ and a $C^{1}$-open set $\mathcal{D}$ of embeddings of $(k-1)$-dimensional disks $D$ into $M$ such that for every $g \in \mathcal{U}$ and every disk $D \in \mathcal{D}$ the local stable manifold $W_{\text {loc }}^{s}\left(\Lambda_{g}\right)$ of $\Lambda_{g}$ (the continuation of $\Lambda$ for $g$ ) intersects $D$. The set $\mathcal{D}$ is called the superposition set of the blender. 
We now give some preliminary definitions that we borrow from [2]. Let $\Delta=$ $I_{x} \times I_{y} \times I_{z}$ be a cube in $\mathbb{R}^{3}$ where $I_{x}=\left[x^{-}, x^{+}\right], I_{y}=\left[y^{-}, y^{+}\right]$and $I_{z}=\left[z^{-}, z^{+}\right]$ are intervals. Divide the boundary of $\Delta$ into three parts as follows:

$$
\partial^{s s} \Delta:=\partial I_{x} \times I_{y} \times I_{z}, \partial^{u u} \Delta:=I_{x} \times \partial I_{y} \times I_{z}, \partial^{u} \Delta:=I_{x} \times \partial\left(I_{y} \times I_{z}\right) .
$$

Note that $\partial^{u u} \Delta \subset \partial^{u} \Delta$. For $\theta>1$ let $\mathcal{C}_{\theta}^{u}, \mathcal{C}_{\theta}^{u u}$, and $\mathcal{C}_{\theta}^{s}$ be cone fields defined as follows: for $p \in \Delta$, put

$$
\begin{aligned}
& \mathcal{C}_{\theta}^{u}(p)=\left\{(u, v, w) \in T_{p} \Delta|\theta| u \mid \leqslant \sqrt{v^{2}+w^{2}}\right\}, \\
& \mathcal{C}_{\theta}^{u u}(p)=\left\{(u, v, w) \in T_{p} \Delta\left|\theta \sqrt{u^{2}+w^{2}} \leqslant\right| v \mid\right\}, \\
& \mathcal{C}_{\theta}^{s}(p)=\left\{(u, v, w) \in T_{p} \Delta\left|\theta \sqrt{v^{2}+w^{2}} \leqslant\right| u \mid\right\} .
\end{aligned}
$$

Note that $\mathcal{C}_{\theta}^{u u}(p) \subset \mathcal{C}_{\theta}^{u}(p)$.

Then we define as follows:

- A regular curve $L \subset \Delta$ is vertical if $T_{p} L \subset \mathcal{C}_{\theta}^{u u}(p)$ for every point $p$ in $L$, and the end-points of $L$ are contained in different connected components of $\partial^{u u} \Delta$.

- A surface $S \subset \Delta$ is called a vertical strip in $\Delta$ if $T_{p} S \subset \mathcal{C}_{\theta}^{u}(p)$ for every $p$ in $S$ and there exists a $C^{1}$ embedding $E: I_{y} \times J \rightarrow \Delta$ (where $J$ is a subinterval of $\left.I_{z}\right)$ such that $L(z):=E\left(I_{y} \times\{z\}\right)$ is a vertical curve for every $z \in J$. The width of $S$, denoted by $w(S)$, is the infimum of the length of the curves in $S$ which are transverse to $\mathcal{C}_{\theta}^{u u}$ joining the two boundary components of $L(\partial J)$.

Let $W$ be a curve in $\Delta$ tangent to the cone field $\mathcal{C}^{s}$ whose endpoints are contained in different connected components of $\partial^{s s} \Delta$. Note that there are two different homotopy classes of vertical segments through $\Delta$ disjoint from $W$.

- A vertical curve $L$ in $\Delta$ with $L \cap W=\emptyset$ is to the right of $W$ if it is in the homotopy class of $\left\{x^{-}\right\} \times I_{y} \times\left\{z^{+}\right\}$for some $x_{0} \in I_{x}$. Similarly, a vertical strip $S$ through $\Delta$ is to the right of $W$ if any vertical curve in $S$ is to the right of $W$.

For a three dimensional diffeomorphism $F$, the next geometric conditions (H1)(H5) guarantee the existence of a blender in $\Delta$, see [2, §1]:

(H1) There is a connected component $A$ of $\Delta \cap F(\Delta)$ disjoint from $\partial^{s s} \Delta \cup F\left(\partial^{u} \Delta\right)$.

(H2) There is a connected component $B$ of $F(\Delta) \cap \Delta$ such that $B$ is disjoint from $I_{x} \times I_{y} \times\left\{z^{+}\right\}$, from $\partial^{s s} \Delta$ and from $F\left(\partial^{u u} \Delta\right)$.

(H3) There are $\theta>1$ and $\ell \in \mathbb{N}$ such that the cone fields $\mathcal{C}_{\theta}^{u}, \mathcal{C}_{\theta}^{u u}$, and $\mathcal{C}_{\theta}^{s}$ satisfy the following conditions: There is $c>1$ such that

(i) For every $p \in F^{-1}(A \cup B)$ such that $F^{i}(p) \in \Delta$ for every $i=0, \ldots, \ell-1$ and every $\mathbf{v} \in \mathcal{C}_{\theta}^{u}(p) \backslash\{\mathbf{0}\},\left(D F^{i}\right)_{p} \mathbf{v}$ belongs to the interior of $\mathcal{C}_{\theta}^{u}\left(F^{i}(p)\right)$ and $\left|\left(D F^{\ell}\right)_{p} \mathbf{v}\right| \geqslant c|\mathbf{v}|$.

(ii) For every $p \in F^{-1}(A \cup B)$ and every $\mathbf{v} \in \mathcal{C}_{\theta}^{u u}(p) \backslash\{\mathbf{0}\}$, w $:=(D F)_{p} \mathbf{v}$ belongs to the interior of $\mathcal{C}_{\theta}^{u u}(F(p))$.

(iii) For every $p \in A \cup B$ and every $\mathbf{v} \in \mathcal{C}_{\theta}^{s}(p) \backslash\{\mathbf{0}\}$, w $:=\left(D F^{-1}\right)_{p} \mathbf{v}$ belongs to the interior of $\mathcal{C}_{\theta}^{s}\left(F^{-1}(p)\right)$ and $|\mathbf{w}| \geqslant c|\mathbf{v}|$.

Note that conditions (H1) and (H3) imply that $F$ has a (unique) hyperbolic fixed point $P_{*}$ in $A$ with index one. Let $W_{0}^{s}$ be the connected component of $W^{s}\left(P_{*}\right) \cap \Delta$ 
containing $P_{*}$. Observe that the curve $W_{0}^{s}$ is tangent to the cone field $\mathcal{C}_{\theta}^{s}$. So we can speak of vertical curves and strips being to the left or to the right of $W_{0}^{s}$.

(H4) There is a neighborhood $U^{-}$of the left side $\left\{z=z^{-}\right\}$of $\Delta$ so that every vertical strip $S$ through $\Delta$ to the right of $W_{0}^{s}$ does not intersect $U^{-}$.

(H5) There exist neighborhoods $U$ of $W_{0}^{s}$ and $U^{+}$of the right side $\left\{z=z^{+}\right\}$of $\Delta$ such that for every vertical strip $S$ through $\Delta$ to the right of $W_{0}^{s}$ one of the two following possibilities holds:

(i) The intersection $F(S) \cap A$ contains a vertical strip $\Sigma$ through $\Delta$ to the right of $W_{0}^{s}$ and disjoint from $U^{+}$;

(ii) $F(S) \cap B$ contains a vertical strip $\Sigma$ through $\Delta$ to the right of $W_{0}^{s}$ and disjoint from $U$.

Note that the presentation of (H3) is slightly different from the one in 2]. In our (H5), each non-zero vector of $\mathcal{C}_{\theta}^{u u}$ is expanded only after $\ell$ iterations. However, by some standard argument, one can check that the conditions (H1)-(H5) above are also sufficient to guarantee the occurrence of the blender. Indeed, these conditions imply that the width of vertical strips in $\Delta$ grows exponentially after iterations by $F^{\ell}$. This implies that the stable manifold $W^{s}\left(P_{*}\right)$ of $P_{*}$ intersects transversally every vertical strip $S$ through $\Delta$ to the right of $W_{0}^{s}$, see [2, Lemma 1.8]. In particular, for $\Lambda=\bigcap_{i \in \mathbb{Z}} F^{i}(\Delta)$ we have $W_{\text {loc }}^{s}(\Lambda) \cap S \neq \emptyset$. Thus $\Lambda$ is a blender whose superposition set is formed by the vertical segments through $\Delta$ to the right of $W_{0}^{s}$.

2.2. Proof of Theorem 1.1. We consider the open set of parameters $\mathcal{O}$ and the cube $\Delta$ in $\mathbb{R}^{3}$ defined as follows:

$$
\begin{aligned}
& \mathcal{O}=\left\{(\mu, \kappa, \xi) \mid-10<\mu<-9,0<\kappa<10^{-4}, 1.18<\xi<1.19\right\}, \\
& \Delta=\{(x, y, z)|| x|,| y \mid \leqslant 4,-40 \leqslant z \leqslant 0\} .
\end{aligned}
$$

The boundary $\partial \Delta$ of $\Delta$ is divided into two parts $\partial^{u} \Delta:=[-4,4] \times \partial([-4,4] \times[-40,0])$ and $\partial^{s s} \Delta:=\Delta \cap\{x= \pm 4\}$. Moreover, consider the subset $\partial^{u u} \Delta:=\Delta \cap\{y= \pm 4\}$ of $\partial^{u} \Delta$. The goal of this section is to prove the following proposition.

Proposition 2.2. There is $\varepsilon>0$ such that every diffeomorphism $F$ sufficiently close to $G_{\mu, \kappa, \xi, \eta}$ (see (1.1) for the definition of $\left.G_{\mu, \kappa, \xi, \eta}\right)$ where $(\mu, \kappa, \xi, \eta) \in \mathcal{O} \times$ $(-\varepsilon, \varepsilon)$ has a blender.

This proposition immediately implies Theorem 1.1. It follows from the next three lemmas and the sufficient conditions for blenders in Section 2.1. So, let us start the proof of the proposition above.

Lemma 2.3. If a three-dimensional diffeomorphism $F$ is sufficiently $C^{1}$-close to $G_{\mu, \kappa, \xi}$ with $(\mu, \kappa, \xi) \in \mathcal{O}$, then there are compact subsets $A$ and $B$ of $\Delta$ satisfying (H1) and (H2) for $F$.

Proof. First, we investigate these properties for $G_{\mu, \kappa, \xi}$ with $(\mu, \kappa, \xi) \in \mathcal{O}$.

Let $\Pi_{z}: \mathbb{R}^{3} \rightarrow \mathbb{R}^{2}$ and $\Pi_{x}: \mathbb{R}^{3} \rightarrow \mathbb{R}^{2}$ be the projections $\Pi_{z}(x, y, z)=(x, y)$ and $\Pi_{x}(x, y, z)=(y, z)$. We first observe the relation between $\Pi_{z}(\Delta)$ and $G_{\mu, \kappa, \xi}(\Delta)$. For $z \in[-40,0]$, write

$$
D_{z}=[-4,4]^{2} \times\{z\}, \quad \partial^{u u} D_{z}=[-4,4] \times\{-4,4\} \times\{z\} .
$$

Note that, $G_{\mu, \kappa, \xi}$ is an endomorphism such that $D G_{\mu, \kappa, \xi}$ has a zero eigenvalue whose eigenspace is the $x$-axis. Thus, for every $z \in[-40,0]$, from (2.1) we have

$$
\Pi_{z}\left(G_{\mu, \kappa, \xi}\left(D_{z}\right)\right)=\left\{(x, y)\left|y=\mu+x^{2}+\kappa z^{2},\right| x \mid \leqslant 4\right\} .
$$


Consider the sets

$$
D_{z}^{+}:=D_{z} \cap\{y>0\}, \quad \text { and } \quad D_{z}^{-}:=D_{z} \cap\{y<0\} .
$$

Since $-10<\mu<-9$ and $0<\kappa z^{2}<0.16$, by direct calculations, one can obtain the following conditions, see Figure 2.1 (a):

- $\Pi_{z}\left(G_{\mu, \kappa, \xi}\left(D_{z}^{+}\right)\right) \cap \Pi_{z}(\Delta)$ contains a segment $I_{z}^{+}$transverse in the $x y$-plane to $\Pi_{z}\left(\partial^{u u} \Delta\right)$ and such that $I_{z}^{+} \subset\{(x, y) \mid 2.4<x<3.8\}$.

- $\Pi_{z}\left(G_{\mu, \kappa, \xi}\left(D_{z}^{-}\right)\right) \cap \Pi_{z}(\Delta)$ contains a segment $I_{z}^{-}$transverse in the $x y$-plane to $\Pi_{z}\left(\partial^{u u} \Delta\right)$ and such that $I_{z}^{-} \subset\{(x, y) \mid-3.8<x<-2.4\}$.

- We have $\Pi_{z}\left(G_{\mu, \kappa, \xi}\left(\partial^{u u} D_{z}\right)\right)=\left\{\left( \pm 4, \mu+16+\kappa z^{2}\right)\right\}$. By the conditions on the constants, its $y$-coordinate $\mu+16+\kappa z^{2}$ is greater than 4 . That is, the projection $\Pi_{z}\left(G_{\mu, \kappa, \xi}\left(\partial^{u u} D_{z}\right)\right)$ is outside $\Pi_{z}(\Delta)$.

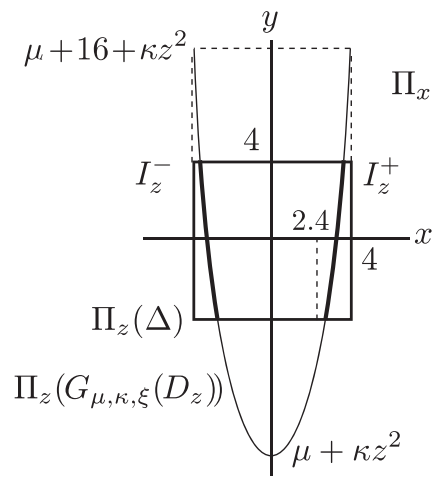

(a)

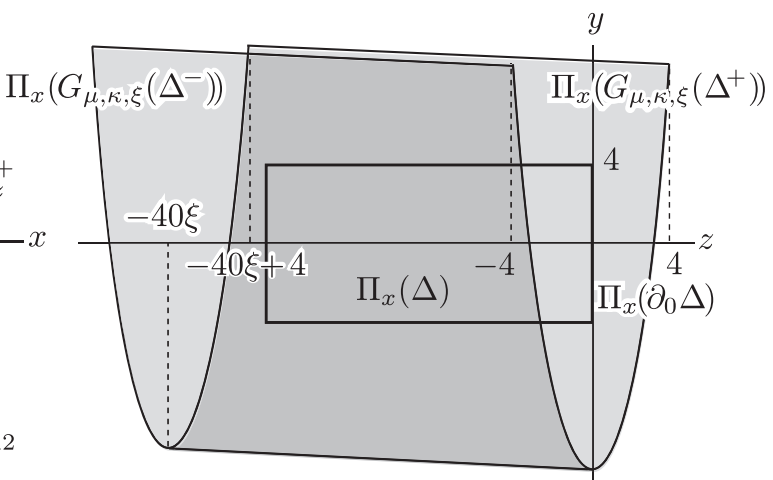

(b)

FIGURE 2.1. Projected images of $\Delta$ and $G_{\mu, \kappa, \xi}(\Delta)$

Consider the sets

$$
\Delta^{+}:=\Delta \cap\{y>0\}, \quad \Delta^{-}:=\Delta \cap\{y<0\}, \quad \text { and } \quad \partial_{0} \Delta:=\Delta \cap\{z=0\} .
$$

The following properties of $\Pi_{x}(\Delta)$ and $\Pi_{x}\left(G_{\mu, \kappa, \xi}(\Delta)\right)$ can be checked by direct calculations, see Figure 2.1. (b).

- $\Pi_{x}\left(G_{\mu, \kappa, \xi}\left(\Delta^{+}\right)\right)$contains $\Pi_{x}(\Delta)=\{(z, y)|-40 \leqslant z \leqslant 0| y \mid, \leqslant 4\}$ in its interior.

- $\Pi_{x}\left(G_{\mu, \kappa, \xi}\left(\Delta^{-}\right)\right) \cap \Pi_{x}(\Delta)$ contains the set $\Pi_{x}(\Delta) \cap\{-40<z<-4\}$, and

Note that

- $\Pi_{x}\left(G_{\mu, \kappa, \xi}\left(\Delta^{ \pm}\right)\right) \cap \Pi_{x}\left(\partial_{0} \Delta\right)=\emptyset$.

Consider now the sets given by

$$
\Delta^{ \pm}=\bigcup_{z \in[-40,0]} D_{z}^{ \pm}
$$

$$
A=G_{\mu, \kappa, \xi}\left(\Delta^{+}\right) \cap \Delta \quad \text { and } \quad B=G_{\mu, \kappa, \xi}\left(\Delta^{-}\right) \cap \Delta .
$$

By the comments above,

$$
\Pi_{z}(A)=\bigcup_{z \in[-40,0]} I_{z}^{+} \quad \text { and } \quad \Pi_{z}(B)=\bigcup_{z \in[-40,0]} I_{z}^{-},
$$

and $\Pi_{x}(A)=\Pi_{x}(\Delta)$ and $\Pi_{x}(B) \cap \Pi_{x}\left(\partial_{0} \Delta\right)=\emptyset$. Moreover, from the above observations, we have 
- $A \cap \partial^{s s} \Delta=\emptyset$ and $A \cap G_{\mu, \kappa, \xi}\left(\partial^{u} \Delta\right)=\emptyset$;

- $B \cap \partial^{s s} \Delta=\emptyset, B \cap \partial_{0} \Delta=\emptyset$, and $B \cap G_{\mu, \kappa, \xi}\left(\partial^{u u} D_{z}\right)=\emptyset$.

This implies that $A$ and $B$ satisfy (H1) and (H2).

Clearly, any diffeomorphism $F$ sufficiently $C^{1}$ close to $G_{\mu, \kappa, \xi}$ also satisfies these properties.

Lemma 2.4. For any diffeomorphism $F$ sufficiently $C^{1}$-close to $G_{\mu, \kappa, \xi},(\mu, \kappa, \xi) \in$ $\mathcal{O}$, the cone fields $\mathcal{C}_{2}^{u}, \mathcal{C}_{2}^{u u}$ and $\mathcal{C}_{2}^{s}$ satisfy (H3) for $A, B$ as in Lemma 2.3.

Proof. First, note that for $p=(x, y, z) \in \Delta$ with $G_{\mu, \kappa, \xi}(p) \in A \cup B$ and $\mathbf{v}=$ $(u, v, w) \in T_{p} \Delta$, we have

$$
\left(u_{1}, v_{1}, w_{1}\right):=\left(D G_{\mu, \kappa, \xi}\right)_{p} \mathbf{v}=(v, 2 y v+2 \kappa z w, v+\xi w) .
$$

For the proof of (H3)-(i) and (ii), we just investigate the property of $G_{\mu, \kappa, \xi}$ with $(\mu, \kappa, \xi) \in \mathcal{O}$, which implies that the same conditions hold for $F$ near $G_{\mu, \kappa, \xi}$.

Proof of (H3)-(i). Consider the cone

$$
\mathcal{C}^{u}(p):=\mathcal{C}_{2}^{u}(p)=\left\{(u, v, w) \in T_{p} \mathbb{R}^{3}|2| u \mid \leqslant \sqrt{v^{2}+w^{2}}\right\} .
$$

We consider the norm

$$
|(u, v, w)|_{*}:=\max \left\{|u|, \sqrt{v^{2}+w^{2}}\right\} .
$$

We will see that $\left|\left(D G_{\mu, \kappa, \xi}\right)_{p} \mathbf{v}\right|_{*}>|\mathbf{v}|_{*}$ for every $\mathbf{v} \in \mathcal{C}^{u u}(p) \backslash\{\mathbf{0}\}$. By compactness this implies that $\left|\left(D G_{\mu, \kappa, \xi}\right)_{p} \mathbf{v}\right|_{*}>c_{0}|\mathbf{v}|_{*}$ for some uniform $c_{0}>1$. Since $|\cdot|_{*}$ is equivalent to $|\cdot|$, this implies that there are $\ell$ and $c>0$ such that $\left|\left(D G_{\mu, \kappa, \xi}^{\ell}\right)_{p} \mathbf{v}\right|>$ $c|\mathbf{v}|$. We now go to the details of the proof.

First, note that from the proof of Lemma 2.3, the condition $G_{\mu, \kappa, \xi}(p) \in A \cup B$ implies $|y|>2.4$. (remember that the $x$-coordinate of $G_{\mu, \kappa, \xi}(p)$ is equal to the $y$-coordinate of $p$ ).

We divide the proof into two cases: $6.5|v| \geqslant|w|$ and $6.5|v|<|w|$. In the case of $6.5|v| \geqslant|w|$, by this inequality and the choice of parameters in 2.4 , we have

$$
\left|v_{1}\right|=|2 y v+2 \kappa z w| \geqslant 2|y||v|-2|\kappa||z||w|>4.8|v|-0.052|v|>4.7|v| .
$$

Thus,

$$
\sqrt{v_{1}^{2}+w_{1}^{2}} \geq\left|v_{1}\right| \geqslant 4.7|v|=4.7\left|u_{1}\right| .
$$

Therefore, $\left(u_{1}, v_{1}, w_{1}\right) \in \mathcal{C}^{u}\left(G_{\mu, \kappa, \xi}(p)\right)$.

To get the uniform expansion of the vectors, note that

$$
w_{1}^{2}=(v+\xi w)^{2} \geqslant v^{2}-2 \xi|v||w|+\xi^{2} w^{2} \geqslant(1-13 \xi) v^{2}+\xi^{2} w^{2},
$$

thus, together with 2.6), one obtains

$$
\left|\left(D G_{\mu, \kappa, \xi}\right)_{p} \mathbf{v}\right|_{*}^{2}=v_{1}^{2}+w_{1}^{2} \geqslant(22-13 \xi) v^{2}+\xi^{2} w^{2}>4 v^{2}+1.18^{2} w^{2}>|\mathbf{v}|_{*}^{2} .
$$

Next, we consider the case of $6.5|v|<|w|$. Since

$$
\left|w_{1}\right|=|v+\xi w| \geqslant \xi|w|-|v| \geqslant 6.5 \xi|v|-|v|>5|v|,
$$

one has

$$
\sqrt{v_{1}^{2}+w_{1}^{2}} \geqslant\left|w_{1}\right|>5|v|=5\left|u_{1}\right|
$$


This implies $\left(u_{1}, v_{1}, w_{1}\right) \in \mathcal{C}^{u}\left(G_{\mu, \kappa, \xi}(p)\right)$. Moreover, by 2.4 , 2.5) and $2.4<|y|<$ 3.8 ,

$$
\begin{aligned}
& v_{1}^{2}=(2 y v+2 \kappa z w)^{2} \geqslant 4 y^{2} v^{2}-8|y v \kappa z w|+4 \kappa^{2} z^{2} w^{2}>23 v^{2}-0.02 w^{2}, \\
& w_{1}^{2}=(v+\xi w)^{2} \geqslant \xi^{2} w^{2}-2 \xi|v||w|+v^{2}>1.026 w^{2}+v^{2} .
\end{aligned}
$$

Therefore,

$$
\left|\left(D G_{\mu, \kappa, \xi}\right)_{p} \mathbf{v}\right|_{*}^{2}=v_{1}^{2}+w_{1}^{2}>24 v^{2}+1.006 w^{2}>|\mathbf{v}|_{*}^{2} .
$$

This completes the proof of (H3)-(i).

Proof of (H3)-(ii). For $p \in \Delta$ with $G_{\mu, \kappa, \xi}(p) \in A \cup B$, take a cone

$$
\mathcal{C}^{u u}(p):=\mathcal{C}_{2}^{u u}(p)=\left\{(u, v, w) \in T_{p} \mathbb{R}^{3}\left|2 \sqrt{u^{2}+w^{2}} \leqslant\right| v \mid\right\} \subset \mathcal{C}^{u}(p) .
$$

This implies that $|w| \leq|v| / 2$ for any $(u, v, w) \in \mathcal{C}^{u u}(p)$. Recalling 2.5 and $2.4<$ $|y|$, we have

$$
\left|v_{1}\right|=|2 y v+2 \kappa z w| \geqslant 2|y||v|-2|\kappa||z||w|>4.7|v| .
$$

On the other hand,

$$
u_{1}^{2}+w_{1}^{2}=v^{2}+(v+\xi w)^{2} \leqslant 2 v^{2}+2 \xi|v||w|+\xi^{2} w^{2} \leqslant 2 v^{2}+1.19 v^{2}+0.63 v^{2}<4 v^{2} .
$$

Hence,

$$
2 \sqrt{u_{1}^{2}+w_{1}^{2}}<4|v|<\left|v_{1}\right| .
$$

Thus $\left(D G_{\mu, \kappa, \xi}\right)_{p} \mathbf{v} \in \mathcal{C}^{u u}\left(G_{\mu, \kappa, \xi}(p)\right)$, which completes the proof of (H3)-(ii).

Proof of (H3)-(iii). The existence of a contracting and invariant strong stable cone field follows from the fact that $D G_{\mu, \kappa, \xi}$ is an endomorphism whose eigenspace associated the eigenvalue 0 is spanned by $(1,0,0)$. This implies that for every diffeomorphism $F$ sufficiently close to $G_{\mu, \kappa, \xi}$ with $(\mu, \kappa, \xi) \in \mathcal{O}$, the cone field $\mathcal{C}_{2}^{s}(p)$ with $p \in A \cup B$ satisfies (H3)-(iii).

Lemma 2.5. Every diffeomorphism $F$ sufficiently $C^{1}$-close to $G_{\mu, \kappa, \xi}$, with $(\mu, \kappa, \xi) \in$ $\mathcal{O}$, satisfies conditions (H4) and (H5) for $A, B$ as in Lemma 2.3 .

Proof. Again, we mainly consider the properties for $G_{\mu, \kappa, \xi}$ with $(\mu, \kappa, \xi) \in \mathcal{O}$. The conclusion for $F$ near $G_{\mu, \kappa, \xi}$ follows almost immediately.

Proof of (H4). By construction, $A$ contains unique saddle fixed point $P_{*}=$ $\left(x_{*}, y_{*}, z_{*}\right)$ which satisfies

$$
x_{*}=y_{*}=\mu+y_{*}^{2}+\kappa z_{*}^{2}=(1-\xi) z_{*} .
$$

Note that $W_{0}^{s}=\left\{\left(x, y_{*}, z_{*}\right)|| x \mid \leq 4\right\}$ and that every point in $W_{0}^{s}$ is mapped to $P_{*}$ by $G_{\mu, \kappa, \xi}$ by $G_{\mu, \kappa, \xi}$. Therefore $W_{0}^{s}$ is a local stable manifold of $P_{*}$. Since $2.4<y_{*}<3.8$ and $1.18<\xi<1.19$, one has the following estimation:

$$
-21.2<z_{*}=-\frac{y_{*}}{\xi-1}<-12.6 .
$$

Note that the cones $\mathcal{C}^{u u}(p)$ are defined around the $y$-axis with slope $1 / 2$. Thus a simple calculation gives that any vertical curve $L$, (i.e., a curve with $T_{p} L \subset \mathcal{C}^{u u}(p)$ ), through $\Delta$ to the right $W_{0}^{s}$ does not intersect a small neighborhood $U^{-}$of $\{z=$ $-40\} \cap \Delta$. This implies that (H4) holds for $G_{\mu, \kappa, \xi}$ and thus for every diffeomorphism $F$ sufficiently $C^{1}$-close to $G_{\mu, \kappa, \xi}$. 
Proof of (H5). Consider the subsets of $\Delta$ defined by

$$
\begin{aligned}
& A^{\prime}=\{(x, y, z)|| x \mid \leqslant 4,2.4 \leqslant y \leqslant 3.8,-22 \leqslant z \leqslant-3.3\}, \\
& B^{\prime}=\{(x, y, z)|| x \mid \leqslant 4,-3.8 \leqslant y \leqslant-2.4,-7.3 \leqslant z \leqslant 0\} .
\end{aligned}
$$

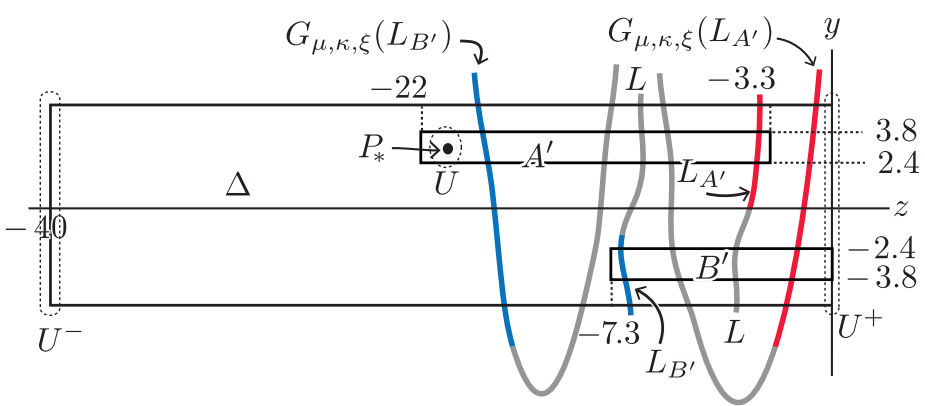

FiguRE 2.2. Vertical segments $L_{A^{\prime}}, L_{B^{\prime}}$ and their images

We define the notion of vertical curves through $A^{\prime}$ (resp. $B^{\prime}$ ) in the natural way. That is, a curve $\sigma$ in $A^{\prime}$ (resp. $B^{\prime}$ ) is called vertical if it is tangent to $\mathcal{C}^{u u}$ and connects $A^{\prime} \cap\{y=3.8\}$ and $A^{\prime} \cap\{y=2.4\}$ (resp. $B^{\prime} \cap\{y=-3.8\}$ and $\left.B^{\prime} \cap\{y=-2.4\}\right)$.

Then, one can observe the following (see Figure 2.2): For every vertical curve $L$ through $\Delta$ to the right of $W_{0}^{s}$,

- either $L_{A^{\prime}}=L \cap A^{\prime}$ is a vertical curve through $A^{\prime}$,

- or $L_{B^{\prime}}=L \cap B^{\prime}$ is a vertical curve through $B^{\prime}$ (note that both cases may hold simultaneously).

In each case, we have the following:

- In the first case, $G_{\mu, \kappa, \xi}\left(L_{A^{\prime}}\right)$ contains a vertical curve through $\Delta$ which is to the right of $W_{0}^{s}$ and disjoint from a small neighborhood $U^{+}$of $\partial_{0} \Delta$ (remember that $\partial_{0} \Delta=\Delta \cap\{z=0\}$ );

- In the second case, $G_{\mu, \kappa, \xi}\left(L_{B^{\prime}}\right)$ contains a vertical curve through $\Delta$ which is to the right of $W_{0}^{s}$ and disjoint from a small neighborhood $U$ of $W_{0}^{s}$.

Therefore, (H5) holds for $G_{\mu, \kappa, \xi}$, and for any diffeomorphism sufficiently $C^{1}$-close to $G_{\mu, \kappa, \xi}$.

Remark 2.6. In the proof above, we can see that the image of the segment $\hat{\ell}:=$ $\{(0, t, 0)|| t \mid<4\}$ under diffeomorphism $F$ which is sufficiently close to $G_{\mu, \kappa, \xi}$ contains a vertical segment which is to the right of $W_{0}^{s}$ of the blender. Thus it has non-empty intersection with the stable manifold of the blender. This fact is used in Section [6.

\section{THE NON-TRANSVERSE HETERODIMENSIONAL CYCLES}

In this section we describe the conditions satisfied by the diffeomorphism with non-transverse heterodimensional cycles in Theorem 1.2 . 
3.1. Local dynamics at the saddle points. Let $P$ and $Q$ be saddle periodic points of $f \in \operatorname{Diff}^{r}(M)$ with $\operatorname{dim}(M)=3$ and $r \geqslant 2$. In what follows, we suppose that $\operatorname{per}(P)=\operatorname{per}(Q)=1$ for simplicity, that the points have indices $\operatorname{index}(P)=2$ and index $(Q)=1$, and that the unstable manifold $W^{u}(P)$ and the stable manifold $W^{s}(Q)$ have a heterodimensional tangency, and the two invariant manifolds $W^{u}(Q)$ and $W^{s}(P)$ have a quasi-transverse intersection. We fix small coordinate neighborhoods $U_{P}$ and $U_{Q}$ of $P$ and $Q$, respectively, and consider transitions between these neighborhoods along the cycle.

For simplicity, we assume that $\left.f\right|_{U_{P}}$ and $\left.f\right|_{U_{Q}}$ are $C^{r}$ linearized, i.e.,

$$
f(x, y, z)= \begin{cases}(\tilde{\lambda} x, \tilde{\sigma} y, \tilde{\zeta} z) & \text { if }(x, y, z) \in U_{P} \\ (\lambda x, \sigma y, \zeta z) & \text { if }(x, y, z) \in U_{Q}\end{cases}
$$

where

$$
|\tilde{\lambda}|<1<|\tilde{\sigma}|<|\tilde{\zeta}|, \quad|\lambda|<|\zeta|<1<|\sigma| .
$$

This assumption is guaranteed by the conditions on the eigenvalues of $D f(P)$ and of $D f(Q)$, see [19, 20]. In what follows we assume that $\lambda$ and $\tilde{\zeta}$ are both positive (in the negative case it is enough to consider a pair number of iterations).

Caveat. In what follows, for simplicity we assume that all eigenvalues are positive. For the cases where some eigenvalues are negative, the proofs work almost similarly with slight modifications.

3.2. Transitions along heteroclinic orbit. In this subsection we describe the transitions from $U_{Q}$ to $U_{P}$ and from $U_{P}$ to $U_{Q}$ along the heteroclinic orbits.

We consider first the transition from $U_{Q}$ to $U_{P}$ along the quasi-transverse orbit $X$. In the linearizing coordinate in $U_{Q}$, up to multiplication by some constant along the the $y$-direction, we can take the quasi-transverse intersection between $W^{u}(Q)$ and $W^{s}(P)$ with $X=(0,1,0) \in U_{Q}$. Similarly, there exists a positive integer $N_{1}$ such that $\tilde{X}=f^{N_{1}}(X)=(1,0,0) \in W_{\text {loc }}^{s}(P)$, see Figure 3.1

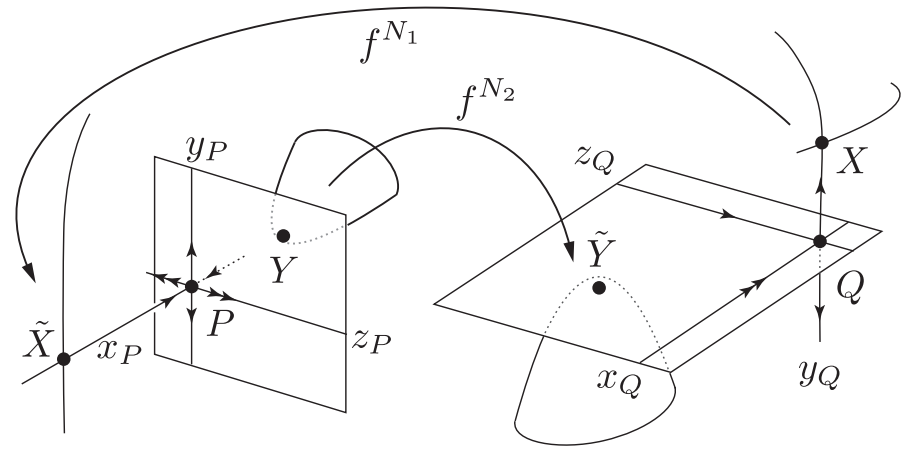

FiguRE 3.1. Non-transverse heterodimensional cycle and its transitions

The transition of $f^{N_{1}}$ from a small neighborhood of $X$ to that of $\tilde{X}$ is expressed as

$$
f^{N_{1}}:\left(\begin{array}{c}
x \\
1+y \\
z
\end{array}\right) \longmapsto\left(\begin{array}{c}
1+\alpha_{1} x+\alpha_{2} y+\alpha_{3} z+\tilde{H}_{1}(x, y, z) \\
\beta_{1} x+\beta_{2} y+\beta_{3} z+\tilde{H}_{2}(x, y, z) \\
\gamma_{1} x+\gamma_{2} y+\gamma_{3} z+\tilde{H}_{3}(x, y, z)
\end{array}\right)
$$


where each $\tilde{H}_{i}$ is the higher order term satisfying the following conditions: for every $i \in\{1,2,3\}$,

$$
\tilde{H}_{i}(\mathbf{0})=0 ;(\partial / \partial x) \tilde{H}_{i}(\mathbf{0})=(\partial / \partial y) \tilde{H}_{i}(\mathbf{0})=(\partial / \partial z) \tilde{H}_{i}(\mathbf{0})=0 .
$$

We assume that

$$
\beta_{3}=\gamma_{2}=\gamma_{3}=0 .
$$

Note that since $f$ is a diffeomorphism,

$$
\beta_{2} \gamma_{1} \neq 0 .
$$

Next, we consider the transition from $U_{P}$ to $U_{Q}$ along the orbit of heterodimensional tangency. Let $Y \in U_{P}$ denote a point of tangency between $W_{\text {loc }}^{u}(P)$ and $W^{s}(Q)$. There is a positive integer $N_{2}$ such that $\tilde{Y}=f^{N_{2}}(Y)$ is contained in $W_{\text {loc }}^{s}(Q)$. By some linear coordinate changes in $U_{P}$ and in $U_{Q}$, one may set $Y=(0,1,1) \in U_{P}$ and $f^{N_{2}}(Y)=(1,0,1) \in U_{Q}$ respectively. Note that this coordinate change can be done independently of the previous one involving $X$ and $X^{\prime}$. Hereafter, these new coordinates are both denoted by $(x, y, z)$.

Since the tangency is nondegenerate, the transition of $f^{N_{2}}$ from a small neighborhood of $Y$ to that of $\tilde{Y}$ is expressed, by taking Taylor expansion, as

$$
f^{N_{2}}:\left(\begin{array}{c}
x \\
1+y \\
1+z
\end{array}\right) \longmapsto\left(\begin{array}{c}
1+a_{1} x+a_{2} y+a_{3} z+H_{1}(x, y, z) \\
b_{1} x+b_{2} y^{2}+b_{3} z^{2}+b_{4} y z+H_{2}(x, y, z) \\
1+c_{1} x+c_{2} y+c_{3} z+H_{3}(x, y, z)
\end{array}\right),
$$

where every $H_{i}$ is the higher order term with the following conditions: for each $i \in\{1,2,3\}$,

$$
H_{i}(\mathbf{0})=0 ;(\partial / \partial x) H_{i}(\mathbf{0})=(\partial / \partial y) H_{i}(\mathbf{0})=(\partial / \partial z) H_{i}(\mathbf{0})=0 .
$$

We assume that

$$
\left(\partial^{2} / \partial y^{2}\right) H_{2}(\mathbf{0})=\left(\partial^{2} / \partial z^{2}\right) H_{2}(\mathbf{0})=\left(\partial^{2} / \partial y \partial z\right) H_{2}(\mathbf{0})=0, \quad c_{3}=0 .
$$

Furthermore, we assume that

$$
b_{2} b_{3} \neq 0, \quad \gamma_{1} a_{3}>0 .
$$

3.3. On the range of eigenvalues. Theorem 1.2 assumes some conditions on the eigenvalues. In this section, we discuss the non-emptiness of the set of the numbers which satisfy these conditions.

We are interested in the existence of 6-ple of numbers

$$
0<\tilde{\lambda}<1<\tilde{\sigma}<\tilde{\zeta}, \quad 0<\lambda<\zeta<1<\sigma
$$

satisfying the following conditions:

$$
\begin{aligned}
& 0<(\tilde{\sigma} \tilde{\zeta})^{k} \sigma \zeta^{2}<1, \\
& 0<\left(\tilde{\sigma}^{-3} \tilde{\zeta}\right)^{k} \sigma^{-1}<1, \\
& 0<(\tilde{\lambda} \tilde{\sigma})^{k} \sigma<1,
\end{aligned}
$$

where $k=\frac{\log \lambda^{-1}}{\log \tilde{\zeta}}$.

We prove the following:

Lemma 3.1. - Let $\mathcal{P} \subset \mathbb{R}^{6}$ denote the set of points $(\tilde{\lambda}, \tilde{\sigma}, \tilde{\zeta}, \lambda, \zeta, \sigma)$ which satisfy the conditions above. Then, $\mathcal{P}$ is a non-empty open set of $\mathbb{R}^{6}$. 
- On $\mathcal{P}$, the value $\log \lambda / \log \zeta$ ranges over $(1,3 / 2)$.

Note that the second item implies that the range of $\alpha$ in Theorem 1.4 is $(0,1 / 2)$.

Proof. The openness of $\mathcal{P}$ is clear. Thus we concentrate on the non-emptiness. There are three inequalities (3.8), (3.9) and (3.10). First, we restrict our attention to $(3.8),(3.9)$. Indeed, if we can prove the non-emptiness of numbers satisfying (3.8) and $(3.9)$, then, by taking sufficiently small $\tilde{\lambda}>0$, we can prove the non-emptiness of the parameter satisfying $(3.10)$.

The inequality (3.8) is equivalent to the following:

$$
\log \sigma<\log \lambda\left(\frac{\log \tilde{\sigma}}{\log \tilde{\zeta}}+1-2 \frac{\log \zeta}{\log \lambda}\right)=: R .
$$

Similarly, by a direct calculation we see that $(3.9)$ is equivalent to

$$
\log \sigma>-\log \lambda\left(1-3 \frac{\log \tilde{\sigma}}{\log \tilde{\zeta}}\right)=: L .
$$

Then, consider the two terms $L$ and $R$. The non-emptiness of $\mathcal{P}$ is equivalent to the following two inequalities:

$$
L<R \text { and } R>0 .
$$

Indeed, if (3.11) holds, then take $\sigma$ so that $\log \sigma>L$ and $\log \sigma<R$ hold (note that the variable $\sigma$ does not appear in $L$ and $R$ and the only restriction on $\sigma$ is $\sigma>1$, that is, $\log \sigma>0$ ).

Put

$$
S:=\frac{\log \tilde{\sigma}}{\log \tilde{\zeta}}, \quad T:=\frac{\log \zeta}{\log \lambda} .
$$

Note that the conditions $1<\tilde{\sigma}<\tilde{\zeta}$ and $1>\zeta>\lambda$ implies that we have

$$
0<S, T<1 .
$$

The inequalities $L<R$ and $R>0$ are respectively equivalent to the following:

$$
T>-S+1 \text { and } T>(1 / 2)(S+1)
$$

By Figure 3.2 below, we know that the set of $(S, T)$ which satisfies these conditions is non-empty.

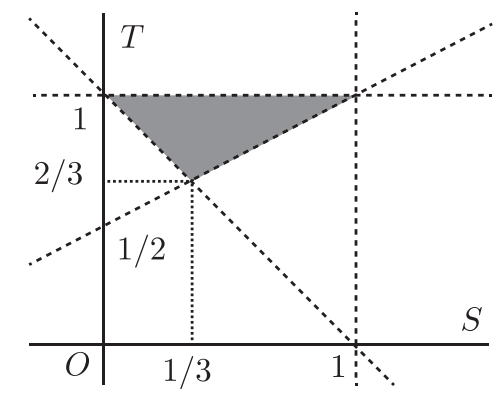

Figure 3.2. The domain of $(S, T)$

Now we prove the non-emptiness of $\mathcal{P}$. First, fix some $(S, T)$ from the region of Figure 3.2 Then, take $(\tilde{\sigma}, \tilde{\zeta})$ and $(\zeta, \lambda)$ which correspond to the values of $(S, T)$. Finally, take $\sigma$ and $\tilde{\lambda}$. This gives us the desired 6-ple of numbers. 
Finally, we see the range of $\frac{\log \lambda}{\log \zeta}=1 / T$. By the figure above, the range of $T$ is $2 / 3<T<1$. Thus the range of $1 / T$ is $(1,3 / 2)$, which completes the proof.

\section{The siX-Parameter family $\left\{f_{\mu, \nu}\right\}$}

In this section we construct a six-parameter family $\left\{f_{\mu, \nu}\right\} \subset \operatorname{Diff}^{r}(M), \mu, \nu \in$ $[-\epsilon, \epsilon]^{3}$, with $f_{\mathbf{0}, \mathbf{0}}=f$ in Theorem 1.2 . This family is obtained by local perturbations near the quasi-transverse intersection $\tilde{X}$ and the heterodimensional tangency $\tilde{Y}$. To define the local perturbations, we use a smooth bump function

$$
B(x, y, z)=b(x) b(y) b(z),
$$

where $b$ is a $C^{r}$ function on $\mathbb{R}$ satisfying

$$
\begin{cases}b(x)=0 & \text { if } 2 \rho \leqslant|x| \\ 0<b(x)<1 & \text { if } \rho<|x|<2 \rho \\ b(x)=1 & \text { if }|x| \leqslant \rho\end{cases}
$$

where $\rho>0$ is some small number. Let $U_{\tilde{X}}$ and $U_{\tilde{Y}}$ be $2 \rho$-neighborhoods of $\tilde{X}=$ $(1,0,0)$ and of $\tilde{Y}=(1,0,1)$ which satisfy $U_{\tilde{X}} \subset U_{P}, P \notin \mathrm{Cl}\left(U_{\tilde{X}}\right)$ and $U_{\tilde{Y}} \subset U_{Q}$, $Q \notin \mathrm{Cl}\left(U_{\tilde{Y}}\right)$, where $\mathrm{Cl}(\cdot)$ means the closure of the corresponding set.

The number $\rho$ is taken so small such that

$$
f\left(U_{\tilde{X}}\right) \cap U_{\tilde{X}}=\emptyset \text { and } f\left(U_{\tilde{Y}}\right) \cap U_{\tilde{Y}}=\emptyset
$$

hold.

For $\mu=\left(\mu_{1}, \mu_{2}, \mu_{3}\right), \nu=\left(\nu_{1}, \nu_{2}, \nu_{3}\right) \in \mathbb{R}^{3}$, let $\left\{t_{\mu, \nu}\right\}$ be a family of maps satisfying the following:

- if $(1+x, y, 1+z) \in U_{\tilde{Y}}$,

$$
t_{\mu, \nu}(1+x, y, 1+z)=(1+x, y, 1+z)+B(x, y, z)\left(\mu_{1}, \mu_{2}, \mu_{3}\right)
$$

- if $(1+x, y, z) \in U_{\tilde{X}}$,

$$
t_{\mu, \nu}(1+x, y, z)=(1+x, y, z)+B(x, y, z)\left(\nu_{1}, \nu_{2}, \nu_{3}\right)
$$

- in the complement of $U_{\tilde{X}} \cup U_{\tilde{Y}}, t_{\mu, \nu}$ is the identity.

Then this family is in $\operatorname{Diff}^{r}(M)$ for small $\mu, \nu \in[-\epsilon, \epsilon]^{3}$ where $\epsilon>0$ is some small number.

We now define

$$
f_{\mu, \nu}=t_{\mu, \nu} \circ f
$$

which has the following properties.

- Since $P \notin \mathrm{Cl}\left(U_{\tilde{X}}\right)$ and $Q \notin \mathrm{Cl}\left(U_{\tilde{Y}}\right), f_{\mu, \nu}$ has saddle periodic points $P_{\mu, \nu}=$ $P$ and $Q_{\mu, \nu}=Q$. Moreover, near $P$ and $Q, f_{\mu, \nu}$ has the same forms as in (3.1).

- For any $(x, 1+y, z)$ sufficiently near $X=(0,1,0)$,

$$
f_{\mu, \nu}^{N_{1}}(x, 1+y, z)=f^{N_{1}}(x, 1+y, z)+\left(\nu_{1}, \nu_{2}, \nu_{3}\right)
$$

which has the same form as $(3.2)$ when $\nu=\mathbf{0}$. The parameters $\nu_{2}$ and $\nu_{3}$ control the unfolding of the quasi-transverse intersection $\tilde{X}$. See Figure 4.1 (a). 
- For any $(x, y+1, z+1)$ sufficiently near $Y=(0,1,1)$,

$$
f_{\mu, \nu}^{N_{2}}(x, 1+y, 1+z)=f^{N_{2}}(x, 1+y, 1+z)+\left(\mu_{1}, \mu_{2}, \mu_{3}\right),
$$

which has the same form as $(3.5)$ when $\mu=\mathbf{0}$. The parameter $\mu_{2}$ controls the unfolding of the heterodimensional tangency at $\tilde{Y}$. See Figure 4.1 (b).

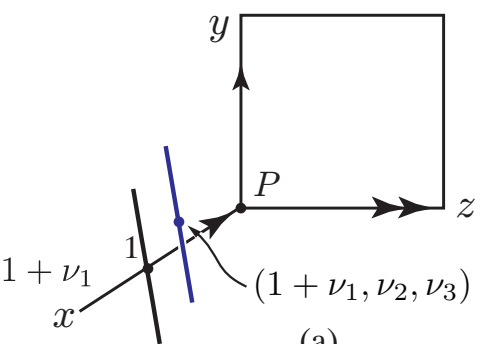

(a)

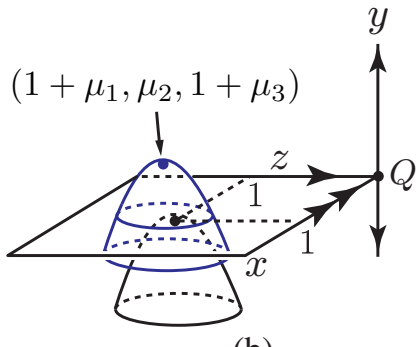

(b)

Figure 4.1. (a) Quasi-transverse intersection (b) heterodimensional tangency

\section{Renormalizations: Proof of Theorem 1.2}

The renormalization scheme involves maps of the form $f_{\mu, \nu}^{N_{2}+m+N_{1}+n}$ where $N_{1}$ and $N_{2}$ are (fixed) transition times, and $n$ and $m$ are the permanence times in $U_{Q}$ and $U_{P}$, respectively. We pick up pair of sojourn times to get return map with neutral behavior.

5.1. For selecting the sojourn times. We first prove an auxiliary result which enables us to get the "neutral dynamics". For that we state an "irrationality condition" of real numbers (Claim 5.2). This condition is used to choose a convenient combination of product of the strong unstable eigenvalue $\tilde{\zeta}$ of $P$ and the strong stable one $\lambda$ of $Q$ in (3.1). With this condition we pick up a sequence of renormalizations converging to a central unstable Hénon-like family.

We put

$$
\mathcal{Z}:=\left\{(\tilde{\zeta}, \lambda) \in \mathbb{R}^{2} \mid 0<\lambda<1<\tilde{\zeta}\right\} .
$$

Lemma 5.1. There is a residual subset $\mathcal{R}$ of $\mathcal{Z}$ such that for every $(\tilde{\zeta}, \lambda) \in \mathcal{R}$ the following holds: For any $\varepsilon>0, N_{0}>0$ and $\xi>0$ satisfying $\varepsilon / \xi<1$, there exist integers $m, n>N_{0}$ such that the following holds:

$$
\left|\gamma_{1} a_{3} \lambda^{n} \tilde{\zeta}^{m}-\xi\right|<\varepsilon, \quad|m-n k-\tilde{k}|<1,
$$

where $\gamma_{1}$ and $a_{3}$ are the constants given in (3.2), (3.5) and (3.7), $k=\log \lambda^{-1} / \log \tilde{\zeta}$ and $\tilde{k}=\log \left(\gamma_{1} a_{3} \xi^{-1}\right) / \log \tilde{\zeta}$.

To prove this lemma we need the following claim whose proof is postponed.

Claim 5.2. There exists a residual subset $\mathcal{R}$ of $\mathcal{Z}$ such that for every $(\tilde{\zeta}, \lambda) \in \mathcal{R}$ the following holds:

$$
\mathrm{Cl}(\{m \log \tilde{\zeta}+n \log \lambda \mid m, n \in \mathbb{N}\})=\mathbb{R} .
$$


Assuming this claim let us give a proof of the lemma above.

Proof of Lemma 5.1. Fix $\varepsilon>0, N_{0}>0$ and $\xi>0$. We take $\varepsilon_{0}>0$ such that $e^{\varepsilon_{0}}-1<\varepsilon / \xi$. By the condition $\varepsilon / \xi<1$, we have $\varepsilon_{0}<1$. From Claim 5.2 and (3.7), for any $\varepsilon_{0}>0, N_{0}>0$ and $\xi>0$, there are integers $m, n>N_{0}$ such that

$$
\left|\log \left(\gamma_{1} a_{3} \xi^{-1}\right)+m \log \tilde{\zeta}+n \log \lambda\right|<\varepsilon_{0} .
$$

For such $m$ and $n$, we have $|m-n k-\tilde{k}|<\varepsilon_{0} / \log \tilde{\zeta}<1$.

By taking the exponential of (5.1), we have

$$
\xi e^{-\varepsilon_{0}}<\gamma_{1} a_{3} \lambda^{n} \tilde{\zeta}^{m}<\xi e^{\varepsilon_{0}} .
$$

Then, by subtracting $\xi$ from each side, we get $\left|\gamma_{1} a_{3} \lambda^{n} \tilde{\zeta}^{m}-\xi\right|<\varepsilon$.

Proof of Claim 5.2. Let $\left\{r_{i}\right\}_{i \in \mathbb{N}}$ be a sequence which is dense in $\mathbb{R}$. For every $r_{i}$ and $j \in \mathbb{N}$, let us consider $U_{i, j} \subset \mathcal{Z}$ defined as follows:

$$
U_{i, j}:=\left\{(\tilde{\zeta}, \lambda) \mid \exists m, n \in \mathbb{N} \text { s.t. }\left|m \log \tilde{\zeta}+n \log \lambda-r_{i}\right|<1 / j\right\} .
$$

The sets $U_{i, j}$ are clearly open in $\mathcal{Z}$. for every positive integers $i$ and $j$. We claim the density of $U_{i, j}$. Then $\mathcal{R}:=\cap_{i, j} U_{i, j}$ is the desired residual set (by Baire's category theorem). Consider the (open) set

$$
V_{i, j}(m, n):=\left\{(X, Y)=(\log \tilde{\zeta}, \log \lambda) \in \mathbb{R}^{2}|| m X+n Y-r_{i} \mid<1 / j\right\} .
$$

Observe that, in $X Y$-plane, $V_{i, j}(m, n)$ is an open strip containing the (open) segment $\left(\left(r_{i}-1 / j\right) / n,\left(r_{i}+1 / j\right) / n\right)$ on the $Y$-axis, going downward right in the fourth quadrant $(0,+\infty) \times(-\infty, 0)$ with slope $-m / n$. Then, by the density of rational numbers $\left\{m / n ; m, n \in \mathbb{Z}_{>0}\right\}$ and taking $n$ large, we get

$$
\mathrm{Cl}\left(\bigcup_{m, n>0} V_{i, j}(m, n)\right)=(0,+\infty) \times(-\infty, 0) \text {. }
$$

Then the pullback of $\cup_{m, n} V_{i, j}(m, n)$ to $\mathcal{Z}$ under the map $(x, y) \mapsto(\log |x|, \log |y|)$ coincides with $U_{i, j}$, which shows the density of $U_{i, j}$.

5.2. Renormalizations near tangencies. Before going our construction recall the conditions $1.3-1.4$ and $3.2-(3.7)$. In what follows we assume that $(\lambda, \tilde{\zeta})$ belongs to the residual subset $\mathcal{R}$ of Lemma 5.1

Our renormalization scheme consists of a sequence of coordinate changes $\Psi_{m, n}$, reparametrizations of $\mu_{m, n}$ and parameters $\nu_{m, n}$ depending on given integers $m, n>$ 0 , which satisfy the following conditions.

- The coordinate change $\Psi_{m, n}: K \rightarrow U_{Q}$, (where $K$ is a compact neighborhood of the origin) $(x, y, z)=\Psi_{m, n}(\bar{x}, \bar{y}, \bar{z})$ is defined by

$$
\Psi_{m, n}(\bar{x}, \bar{y}, \bar{z}):=\left(\sigma^{-n} \tilde{\sigma}^{-m} \bar{x}+1, \sigma^{-2 n} \tilde{\sigma}^{-2 m} \bar{y}+\sigma^{-n}, \sigma^{-n} \tilde{\sigma}^{-m} \bar{z}+1\right) .
$$

Note that $\Psi_{m, n}(K)$ converges to the point of heterodimensional tangency $\tilde{Y}=(1,0,1) \in U_{Q}$ as $m, n \rightarrow \infty$. This means that for any $K$ if $m, n$ are sufficiently large, we can define $\Psi_{m, n}$.

- The reparametrization $\mu_{m, n}: I \rightarrow \mathbb{R}^{3}, \mu=\mu_{m, n}(\bar{\mu})$, is defined by

$$
\mu_{m, n}(\bar{\mu}):=\left(-\tilde{\lambda}^{m} a_{1}, \sigma^{-2 n} \tilde{\sigma}^{-2 m} \bar{\mu}+\sigma^{-n}-\tilde{\lambda}^{m} b_{1},-\tilde{\lambda}^{m} c_{1}\right),
$$

which converges to $\mathbf{0}$ as $m, n \rightarrow \infty$ where $I$ is a (fixed) closed interval. 
- The sequence of parameter values $\left\{\nu_{m, n}\right\}$ is given as

$$
\nu_{m, n}:=\left(-\lambda^{n} \alpha_{1}-\zeta^{n} \alpha_{3}, \tilde{\sigma}^{-m}-\lambda^{n} \beta_{1}, \tilde{\zeta}^{-m}-\lambda^{n} \gamma_{1}\right),
$$

which converges to $\mathbf{0}$ as $m, n \rightarrow \infty$.

Using the renormalization $\left(\Psi_{m, n}, \mu_{m, n}, \nu_{m, n}\right)$, the return map by iterations of $f_{\mu, \nu}$ near the heterodimensional tangency $\tilde{Y}$ is defined by

$$
F_{m, n}(\bar{x}, \bar{y}, \bar{z}):=\left(\left.\Psi_{m, n}^{-1}\right|_{\Psi_{m, n}(K)}\right) \circ f_{\mu_{m, n}(\bar{\mu}), \nu_{m, n}}^{N_{2}+m+N_{1}+n} \circ \Psi_{m, n}(\bar{x}, \bar{y}, \bar{z}),
$$

where $N_{1}$ and $N_{2}$ are the constants in (3.2) and (3.5), which are independent of $m, n$. Note that the domain of $F_{m, n}$ is $K$ and $K$ can be chosen arbitrarily large by letting $m, n$ large.

5.3. Proof of Theorem 1.2. In this subsection, we prove Theorem 1.2, For that we fix $\xi>0$ and select a sequence $\left(m_{k}, n_{k}\right) \subset \mathbb{N}^{2}$ such that $\gamma_{1} a_{3} \lambda^{n_{k}} \widetilde{\zeta}^{m_{k}}$ converges to $\xi$ (recall Lemma 5.1). Then for those $\left\{F_{m_{k}, n_{k}}\right\}$ we get the convergence to the center unstable Hénon-like family.

Proof of (1). The claim is obtained immediately from (5.2)-(5.4) under the conditions for eigenvalues in 1.3 .

Proof of (2). The proof is done by calculating entries in the formula (5.5) using 1.3, 1.4, 3.2 3.7 and $(\lambda, \tilde{\zeta}) \in \mathcal{R}$ of Lemma 5.1 In the proof, we omit the subscript $k$ for simplicity. Thus, for example, we write $F_{m, n}$ in the sense of $F_{m_{k}, n_{k}}$.

Let us provide step-by-step calculations to obtain entries in the formula of return map $F_{m, n}$. By the coordinate change $\Psi_{m, n}$ of $(5.2)$, each $(\bar{x}, \bar{y}, \bar{z}) \in \mathbb{R}^{3}$ is mapped to

$$
\mathbf{x}_{0}:=\left(\sigma^{-n} \tilde{\sigma}^{-m} \bar{x}+1, \sigma^{-2 n} \tilde{\sigma}^{-2 m} \bar{y}+\sigma^{-n}, \sigma^{-n} \tilde{\sigma}^{-m} \bar{z}+1\right) .
$$

Note that if $(\bar{x}, \bar{y}, \bar{z})$ is contained in a compact domain and $m, n$ are sufficiently large, $\mathbf{x}_{0}$ is close to the heterodimensional tangency $\tilde{Y}=(1,0,1)$.

First, after $n$ iterations of $f_{\mu_{m, n}(\bar{\mu}), \nu_{m, n}}$, in other words, the linear transformation (3.1), $\mathbf{x}_{0}$ moves to $f_{\mu_{m, n}(\bar{\mu}), \nu_{m, n}}^{n}\left(\mathbf{x}_{0}\right)=\left(x_{n}, y_{n}+1, z_{n}\right)$ where

$$
\left(x_{n}, y_{n}+1, z_{n}\right)=\left(\lambda^{n} \sigma^{-n} \tilde{\sigma}^{-m} \bar{x}+\lambda^{n}, \sigma^{-n} \tilde{\sigma}^{-2 m} \bar{y}+1, \zeta^{n} \sigma^{-n} \tilde{\sigma}^{-m} \bar{z}+\zeta^{n}\right) .
$$

We write $\tilde{\mathbf{x}}_{n}=\left(x_{n}, y_{n}, z_{n}\right)$. These entries can be described by Landau notation as

$$
x_{n}=O\left(\lambda^{n}\right), y_{n}=O\left(\sigma^{-n} \tilde{\sigma}^{-2 m}\right), z_{n}=O\left(\zeta^{n}\right) .
$$

Note that this also implies that the point $\left(x_{n}, y_{n}+1, z_{n}\right)$ converges to $(0,1,0)$ when $n$ tends to $+\infty$. This guarantees that the points in $\Psi_{m, n}(\Delta)$ stay in $U_{Q}$ and then leave it by the transition map.

Thus, we apply the transition $f_{\mu_{m, n}(\bar{\mu}), \nu_{m, n}}^{N_{1}}$ defined by 4.3 (where the parameter $\mu_{m, n}(\bar{\mu})$ is not yet involved). Recalling the choice of $\nu$ in (5.4) and the conditions $\beta_{3}=\gamma_{2}=\gamma_{3}=0$ in 3.3 we get the following formula in the local coordinate of $U_{P}$,

$f_{\mu_{m, n}(\bar{\mu}), \nu_{m, n}}^{N_{1}+n}\left(\mathbf{x}_{0}\right)=\left(1+\lambda^{n} \sigma^{-n} \tilde{\sigma}^{-m} \alpha_{1} \bar{x}+\sigma^{-n} \tilde{\sigma}^{-2 m} \alpha_{2} \bar{y}+\zeta^{n} \sigma^{-n} \tilde{\sigma}^{-m} \alpha_{3} \bar{z}+\tilde{H}_{1}\left(\tilde{\mathbf{x}}_{n}\right)\right.$, $\left.\tilde{\sigma}^{-m}+\lambda^{n} \sigma^{-n} \tilde{\sigma}^{-m} \beta_{1} \bar{x}+\sigma^{-n} \tilde{\sigma}^{-2 m} \beta_{2} \bar{y}+\tilde{H}_{2}\left(\tilde{\mathbf{x}}_{n}\right), \tilde{\zeta}^{-m}+\lambda^{n} \sigma^{-n} \tilde{\sigma}^{-m} \gamma_{1} \bar{x}+\tilde{H}_{3}\left(\tilde{\mathbf{x}}_{n}\right)\right)$.

Note that from (3.2), for each $i=1,2,3, \tilde{H}_{i}$ starts from a quadratic term. Since $0<\lambda<\zeta$, the dominant terms in $\tilde{H}_{i}$ are $y_{n}=O\left(\sigma^{-n} \tilde{\sigma}^{-2 m}\right)$ and $z_{n}=O\left(\zeta^{n}\right)$ in 
5.6. In fact,

$$
\tilde{H}_{i}\left(\tilde{\mathbf{x}}_{n}\right)=O\left(\sigma^{-2 n} \tilde{\sigma}^{-4 m}\right)+O\left(\zeta^{2 n}\right) .
$$

By the next $m$ iterations of the linear transformation by (3.1) in $U_{P}$, we have $f_{\mu, \nu_{m, n}}^{m+N_{1}+n}\left(\mathbf{x}_{0}\right)=\left(x_{m}, 1+y_{m}, 1+z_{m}\right)$ near $Y=(0,1,1)$ where

$$
\begin{aligned}
& x_{m}=\tilde{\lambda}^{m}+\lambda^{n} \tilde{\lambda}^{m} \sigma^{-n} \tilde{\sigma}^{-m} \alpha_{1} \bar{x}+\tilde{\lambda}^{m} \sigma^{-n} \tilde{\sigma}^{-2 m} \alpha_{2} \bar{y}+\tilde{\lambda}^{m} \sigma^{-n} \tilde{\sigma}^{-m} \zeta^{n} \alpha_{3} \bar{z}+\tilde{\lambda}^{m} \tilde{H}_{1}\left(\tilde{\mathbf{x}}_{n}\right), \\
& 1+y_{m}=1+\lambda^{n} \sigma^{-n} \beta_{1} \bar{x}+\sigma^{-n} \tilde{\sigma}^{-m} \beta_{2} \bar{y}+\tilde{\sigma}^{m} \tilde{H}_{2}\left(\tilde{\mathbf{x}}_{n}\right), \\
& 1+z_{m}=1+\lambda^{n} \sigma^{-n} \tilde{\sigma}^{-m} \tilde{\zeta}^{m} \gamma_{1} \bar{x}+\tilde{\zeta}^{m} \tilde{H}_{3}\left(\tilde{\mathbf{x}}_{n}\right) .
\end{aligned}
$$

Put $\mathbf{x}_{m}:=\left(x_{m}, y_{m}, z_{m}\right)$. From (1.4), (5.6) and (5.7), we have the following:

$$
x_{m}=O\left(\tilde{\lambda}^{m}\right), y_{m}=O\left(\sigma^{-2 n} \tilde{\sigma}^{-3 m}\right)+O\left(\tilde{\sigma}^{m} \zeta^{2 n}\right), z_{m}=O\left(\sigma^{-n} \tilde{\sigma}^{-m}\right) .
$$

We can see that these three numbers converge to zero as $m, n \rightarrow+\infty$. Indeed, the convergence of $x_{m}$ and $z_{m}$ are easy. The convergence of $y_{m}$ comes from the first condition of the eigenvalue condition (1.4), This means that after $m$-times iteration, the point leaves $U_{P}$ by the transition map.

By the transition $f_{\mu_{m, n}(\bar{\mu}), \nu_{m, n}}^{N_{2}}$ (which does not depend on $\left.\nu_{m, n}\right)$, we have in the local coordinate $(\hat{x}, \hat{y}, \hat{z})=f_{\mu_{m, n}(\bar{\mu}), \nu_{m, n}}^{N_{2}+m+N_{1}+n}\left(\mathbf{x}_{0}\right)$ in $U_{Q}$, where

$$
\begin{aligned}
\hat{x}=1 & +\left(\lambda^{n} \tilde{\lambda}^{m} \sigma^{-n} \tilde{\sigma}^{-m} a_{1} \alpha_{1}+\lambda^{n} \sigma^{-n} a_{2} \beta_{1}+\lambda^{n} \sigma^{-n} \tilde{\sigma}^{-m} \tilde{\zeta}^{m} a_{3} \gamma_{1}\right) \bar{x} \\
& +\left(\tilde{\lambda}^{m} \sigma^{-n} \tilde{\sigma}^{-2 m} a_{1} \alpha_{2}+\sigma^{-n} \tilde{\sigma}^{-m} a_{2} \beta_{2}\right) \bar{y}+\tilde{\lambda}^{m} \sigma^{-n} \tilde{\sigma}^{-m} \zeta^{n} a_{1} \alpha_{3} \bar{z} \\
& +\tilde{\lambda}^{m} a_{1} \tilde{H}_{1}\left(\tilde{\mathbf{x}}_{n}\right)+\tilde{\sigma}^{m} a_{2} \tilde{H}_{2}\left(\tilde{\mathbf{x}}_{n}\right)+\tilde{\zeta}^{m} a_{3} \tilde{H}_{3}\left(\tilde{\mathbf{x}}_{n}\right)+H_{1}\left(\mathbf{x}_{m}\right), \\
\hat{y}= & \sigma^{-2 n} \tilde{\sigma}^{-2 m} \bar{\mu}+\sigma^{-n} \\
& +\lambda^{n} \tilde{\lambda}^{m} \sigma^{-n} \tilde{\sigma}^{-m} b_{1} \alpha_{1} \bar{x}+\tilde{\lambda}^{m} \sigma^{-n} \tilde{\sigma}^{-2 m} b_{1} \alpha_{2} \bar{y}+\tilde{\lambda}^{m} \sigma^{-n} \tilde{\sigma}^{-m} \zeta^{n} b_{1} \alpha_{3} \bar{z} \\
& +\left(\lambda^{2 n} \sigma^{-2 n} \beta_{1}^{2} b_{2}+\lambda^{2 n} \sigma^{-2 n} \tilde{\sigma}^{-2 m} \tilde{\zeta}^{2 m} \gamma_{1}^{2} b_{3}+\lambda^{2 n} \sigma^{-2 n} \tilde{\sigma}^{-m} \tilde{\zeta}^{m} \beta_{1} \gamma_{1} b_{4}\right) \bar{x}^{2} \\
& +\sigma^{-2 n} \tilde{\sigma}^{-2 m} \beta_{2}^{2} b_{2} \bar{y}^{2}+\left(2 \lambda^{n} \sigma^{-2 n} \tilde{\sigma}^{-m} \beta_{1} \beta_{2} b_{2}+\lambda^{n} \sigma^{-2 n} \tilde{\sigma}^{-2 m} \tilde{\zeta}^{m} \beta_{2} \gamma_{1} b_{4}\right) \bar{x} \bar{y} \\
& +\tilde{\lambda}^{m} b_{1} \tilde{H}_{1}\left(\tilde{\mathbf{x}}_{n}\right)+\left(2 \lambda^{n} \sigma^{-n} \tilde{\sigma}^{m} \beta_{1} b_{2} \bar{x}+\lambda^{n} \sigma^{-n} \tilde{\zeta}^{m} \gamma_{1} b_{4} \bar{x}+2 \sigma^{-n} \beta_{2} b_{2} \bar{y}\right) \tilde{H}_{2}\left(\tilde{\mathbf{x}}_{n}\right) \\
& +\left(\lambda^{n} \sigma^{-n} \tilde{\zeta}^{m} \beta_{1} b_{4} \bar{x}+2 \lambda^{n} \sigma^{-n} \tilde{\sigma}^{-m} \tilde{\zeta}^{2 m} \gamma_{1} b_{3} \bar{x}+\sigma^{-n} \tilde{\sigma}^{-m} \tilde{\zeta}^{m} \beta_{2} b_{4} \bar{y}\right) \tilde{H}_{3}\left(\tilde{\mathbf{x}}_{n}\right) \\
& +\tilde{\sigma}^{2 m} b_{2} \tilde{H}_{2}\left(\tilde{\mathbf{x}}_{n}\right)^{2}+\tilde{\sigma}^{m} b_{4} \tilde{\zeta}^{m} \tilde{H}_{2}\left(\tilde{\mathbf{x}}_{n}\right) \tilde{H}_{3}\left(\tilde{\mathbf{x}}_{n}\right)+\tilde{\zeta}^{2 m} b_{3} \tilde{H}_{3}\left(\tilde{\mathbf{x}}_{n}\right)^{2}+H_{2}\left(\mathbf{x}_{m}\right), \\
\hat{z}=1 & +\left(\lambda^{n} \tilde{\lambda}^{m} \sigma^{-n} \tilde{\sigma}^{-m} c_{1} \alpha_{1}+\lambda^{n} \sigma^{-n} c_{2} \beta_{1}\right) \bar{x} \\
& +\left(\tilde{\lambda}^{m} \sigma^{-n} \tilde{\sigma}^{-2 m} c_{1} \alpha_{2}+\sigma^{-n} \tilde{\sigma}^{-m} c_{2} \beta_{2}\right) \bar{y}+\tilde{\lambda}^{m} \sigma^{-n} \tilde{\sigma}^{-m} \zeta^{n} c_{1} \alpha_{3} \bar{z} \\
& +\tilde{\lambda}^{m} c_{1} \tilde{H}_{1}\left(\tilde{\mathbf{x}}_{n}\right)+\tilde{\sigma}^{m} c_{2} \tilde{H}_{2}\left(\tilde{\mathbf{x}}_{n}\right)+H_{3}\left(\mathbf{x}_{m}\right) .
\end{aligned}
$$

Finally, using the inverse of $(5.2)$, we get that the return map $F_{m, n}(\bar{x}, \bar{y}, \bar{z})=$ $\left(\bar{x}_{1}, \bar{y}_{1}, \bar{z}_{1}\right)$ defined by 5.5 satisfies

$$
\begin{aligned}
\bar{x}_{1} & =\left(\lambda^{n} \tilde{\lambda}^{m} a_{1} \alpha_{1}+\lambda^{n} \tilde{\sigma}^{m} a_{2} \beta_{1}+\lambda^{n} \tilde{\zeta}^{m} a_{3} \gamma_{1}\right) \bar{x}+\left(\tilde{\lambda}^{m} \tilde{\sigma}^{-m} a_{1} \alpha_{2}+a_{2} \beta_{2}\right) \bar{y} \\
& +\tilde{\lambda}^{m} \zeta^{n} a_{1} \alpha_{3} \bar{z}+\tilde{\lambda}^{m} \sigma^{n} \tilde{\sigma}^{m} a_{1} \tilde{H}_{1}\left(\tilde{\mathbf{x}}_{n}\right)+\sigma^{n} \tilde{\sigma}^{2 m} a_{2} \tilde{H}_{2}\left(\tilde{\mathbf{x}}_{n}\right) \\
& +\sigma^{n} \tilde{\sigma}^{m} \tilde{\zeta}^{m} a_{3} \tilde{H}_{3}\left(\tilde{\mathbf{x}}_{n}\right)+\sigma^{n} \tilde{\sigma}^{m} H_{1}\left(\mathbf{x}_{m}\right),
\end{aligned}
$$


BLENDERS, CU-HÉNON-LIKE FAMILIES AND HETERODIMENSIONAL BIFURCATIONS 21

$$
\begin{aligned}
\bar{y}_{1} & =\bar{\mu}+\lambda^{n} \tilde{\lambda}^{m} \sigma^{n} \tilde{\sigma}^{m} b_{1} \alpha_{1} \bar{x}+\tilde{\lambda}^{m} \sigma^{n} b_{1} \alpha_{2} \bar{y}+\tilde{\lambda}^{m} \sigma^{n} \tilde{\sigma}^{m} \zeta^{n} b_{1} \alpha_{3} \bar{z} \\
& +\left(\lambda^{2 n} \tilde{\sigma}^{2 m} \beta_{1}^{2} b_{2}+\lambda^{2 n} \tilde{\zeta}^{2 m} \gamma_{1}^{2} b_{3}+\lambda^{2 n} \tilde{\sigma}^{m} \tilde{\zeta}^{m} \beta_{1} \gamma_{1} b_{4}\right) \bar{x}^{2}+\beta_{2}^{2} b_{2} \bar{y}^{2} \\
& +\left(2 \lambda^{n} \tilde{\sigma}^{m} \beta_{1} \beta_{2} b_{2}+\lambda^{n} \tilde{\zeta}^{m} \beta_{2} \gamma_{1} b_{4}\right) \bar{x} \bar{y}+\tilde{\lambda}^{m} \sigma^{2 n} \tilde{\sigma}^{2 m} b_{1} \tilde{H}_{1}\left(\tilde{\mathbf{x}}_{n}\right) \\
& +\left(2 \lambda^{n} \sigma^{n} \tilde{\sigma}^{3 m} \beta_{1} b_{2} \bar{x}+\lambda^{n} \sigma^{n} \tilde{\sigma}^{2 m} \tilde{\zeta}^{m} \gamma_{1} b_{4} \bar{x}+2 \sigma^{n} \tilde{\sigma}^{2 m} \beta_{2} b_{2} \bar{y}\right) \tilde{H}_{2}\left(\tilde{\mathbf{x}}_{n}\right) \\
& +\left(2 \lambda^{n} \sigma^{n} \tilde{\sigma}^{m} \tilde{\zeta}^{2 m} \gamma_{1} b_{3} \bar{x}+\lambda^{n} \sigma^{n} \tilde{\sigma}^{2 m} \tilde{\zeta}^{m} \beta_{1} b_{4} \bar{x}+\sigma^{n} \tilde{\sigma}^{m} \tilde{\zeta}^{m} \beta_{2} b_{4} \bar{y}\right) \tilde{H}_{3}\left(\tilde{\mathbf{x}}_{n}\right) \\
& +\sigma^{2 n} \tilde{\sigma}^{4 m} b_{2} \tilde{H}_{2}\left(\tilde{\mathbf{x}}_{n}\right)^{2}+\sigma^{2 n} \tilde{\sigma}^{3 m} \tilde{\zeta}^{m} b_{4} \tilde{H}_{2}\left(\tilde{\mathbf{x}}_{n}\right) \tilde{H}_{3}\left(\tilde{\mathbf{x}}_{n}\right) \\
& +\sigma^{2 n} \tilde{\sigma}^{2 m} \tilde{\zeta}^{2 m} b_{3} \tilde{H}_{3}\left(\tilde{\mathbf{x}}_{n}\right)^{2}+\sigma^{2 n} \tilde{\sigma}^{2 m} H_{2}\left(\mathbf{x}_{m}\right), \\
\bar{z}_{1} & =\left(\lambda^{n} \tilde{\lambda}^{m} c_{1} \alpha_{1}+\lambda^{n} \tilde{\sigma}^{m} c_{2} \beta_{1}\right) \bar{x}+\left(\tilde{\lambda}^{m} \tilde{\sigma}^{-m} c_{1} \alpha_{2}+c_{2} \beta_{2}\right) \bar{y}+\tilde{\lambda}^{m} \zeta^{n} c_{1} \alpha_{3} \bar{z} \\
& +\tilde{\lambda}^{m} \sigma^{n} \tilde{\sigma}^{m} c_{1} \tilde{H}_{1}\left(\tilde{\mathbf{x}}_{n}\right)+\sigma^{n} \tilde{\sigma}^{2 m} c_{2} \tilde{H}_{2}\left(\tilde{\mathbf{x}}_{n}\right)+\sigma^{n} \tilde{\sigma}^{m} H_{3}\left(\mathbf{x}_{m}\right) .
\end{aligned}
$$

Now, we check the convergence of (5.9)-(5.11) as $m, n$ tend to infinity. First, we estimate the higher order terms containing $H_{i}$ and $H_{i}$.

- Higher order terms containing $\tilde{H}_{1}, \tilde{H}_{2}, \tilde{H}_{3}$. The coordinate $\bar{x}_{1}$ in 5.9 has three higher order terms containing $\tilde{H}_{1}, \tilde{H}_{2}$ and $\tilde{H}_{3}$ whose $(m, n)$-dependent coefficients are respectively

$$
\tilde{\lambda}^{m} \sigma^{n} \tilde{\sigma}^{m}, \sigma^{n} \tilde{\sigma}^{2 m}, \sigma^{n} \tilde{\sigma}^{m} \tilde{\zeta}^{m} .
$$

By condition 1.3 , we have

$$
0<\tilde{\lambda}^{m} \sigma^{n} \tilde{\sigma}^{m}, \sigma^{n} \tilde{\sigma}^{2 m}<\sigma^{n} \tilde{\sigma}^{m} \tilde{\zeta}^{m} .
$$

As noted above, every $\tilde{H}_{i}$ has order at least two. Thus, it is enough to check the convergence to 0 of $\sigma^{n} \tilde{\sigma}^{m} \tilde{\zeta}^{m} \tilde{H}_{i}\left(\tilde{\mathbf{x}}_{n}\right)$. From (5.7),

$$
\sigma^{n} \tilde{\sigma}^{m} \tilde{\zeta}^{m} \tilde{H}_{i}\left(\tilde{\mathbf{x}}_{n}\right)=O\left(\sigma^{n} \tilde{\sigma}^{m} \tilde{\zeta}^{m} \zeta^{2 n}\right)+O\left(\sigma^{-n} \tilde{\sigma}^{-3 m} \tilde{\zeta}^{m}\right) .
$$

Since $|m-n k-\tilde{k}|<1$ by Lemma 5.1 , we have

$$
\sigma^{n} \tilde{\sigma}^{m} \tilde{\zeta}^{m} \zeta^{2 n}<C\left(\sigma \tilde{\sigma}^{k} \tilde{\zeta}^{k} \zeta^{2}\right)^{n}, \sigma^{-n} \tilde{\sigma}^{-3 m} \tilde{\zeta}^{m}<C^{\prime}\left(\sigma^{-1} \tilde{\sigma}^{-3 k} \tilde{\zeta}^{k}\right)^{n}
$$

where $C, C^{\prime}>0$ are constants independent of $m$ and $n$. Conditions 1.4 implies that the expression in (5.13) converges to 0 as $m, n \rightarrow+\infty$.

Moreover, using (1.3) and (5.7), one can easily check that the $r$ th order derivatives of $\sigma^{n} \tilde{\sigma}^{m} \tilde{\zeta}^{m} \tilde{H}_{i}\left(\tilde{\mathbf{x}}_{n}\right)$ also converge to 0 as $m, n \rightarrow+\infty$. Thus, these higher order terms also converge to 0 as $m, n \rightarrow+\infty$ in the $C^{r}$ topology on compact domains.

We now evaluate the expression of $\bar{y}_{1}$ in 5.10 , which has higher order terms containing $\tilde{H}_{1}, \tilde{H}_{2}, \tilde{H}_{3}, \tilde{H}_{2}^{2}, \tilde{H}_{2} \tilde{H}_{3}, \tilde{H}_{3}^{2}$. Thus, let us see the convergences of terms containing $\tilde{H}_{1}, \tilde{H}_{2}$ and $\tilde{H}_{3}$. It is enough to study the following $(m, n)$-dependent terms

$$
\tilde{\lambda}^{m} \sigma^{2 n} \tilde{\sigma}^{2 m} \tilde{H}_{i}, \lambda^{n} \sigma^{n} \tilde{\sigma}^{3 m} \tilde{H}_{i}, \sigma^{n} \tilde{\sigma}^{2 m} \tilde{H}_{i}, \lambda^{n} \sigma^{n} \tilde{\sigma}^{m} \tilde{\zeta}^{2 m} \tilde{H}_{i} .
$$

Note that the estimate of $\sigma^{n} \tilde{\sigma}^{2 m} \tilde{H}_{i}$ was already done, see 5.12 . Moreover, since $\lambda^{n} \sigma^{n} \tilde{\sigma}^{m} \tilde{\zeta}^{2 m}=\left(\lambda^{n} \tilde{\zeta}^{m}\right) \sigma^{n} \tilde{\sigma}^{m} \tilde{\zeta}^{m}$ and $\lambda^{n} \tilde{\zeta}^{m}$ converges to a constant by Lemma 5.1 . the convergence of $\lambda^{n} \sigma^{n} \tilde{\sigma}^{m} \tilde{\zeta}^{2 m} \tilde{H}_{i}$ follows from the discussion above, see 5.13 . So we have only to check the convergence of $\tilde{\lambda}^{m} \sigma^{2 n} \tilde{\sigma}^{2 m} \tilde{H}_{1}\left(\tilde{\mathbf{x}}_{n}\right)$ and $\lambda^{n} \sigma^{n} \tilde{\sigma}^{3 m} \tilde{H}_{2}\left(\tilde{\mathbf{x}}_{n}\right)$. By (5.7),

$$
\begin{aligned}
& \tilde{\lambda}^{m} \sigma^{2 n} \tilde{\sigma}^{2 m} \tilde{H}_{1}\left(\tilde{\mathbf{x}}_{n}\right)=O\left(\tilde{\lambda}^{m} \tilde{\sigma}^{-2 m}\right)+O\left(\tilde{\lambda}^{m} \sigma^{2 n} \tilde{\sigma}^{2 m} \zeta^{2 n}\right), \\
& \lambda^{n} \sigma^{n} \tilde{\sigma}^{3 m} \tilde{H}_{2}\left(\tilde{\mathbf{x}}_{n}\right)=O\left(\lambda^{n} \sigma^{-n} \tilde{\sigma}^{-m}\right)+O\left(\lambda^{n} \sigma^{n} \tilde{\sigma}^{3 m} \zeta^{2 n}\right) .
\end{aligned}
$$


By a similar discussion as above, using $|m-n k-\tilde{k}|<1$ of Lemma 5.1 and 1.4 , these terms also converge to 0 as $m, n \rightarrow+\infty$.

Moreover, same as in the case of $\bar{x}_{1}$, one can check the convergence of $r$ th order derivatives of $\tilde{\lambda}^{m} \sigma^{2 n} \tilde{\sigma}^{2 m} \tilde{H}_{1}\left(\tilde{\mathbf{x}}_{n}\right)$ and $\lambda^{n} \sigma^{n} \tilde{\sigma}^{3 m} \tilde{H}_{2}\left(\tilde{\mathbf{x}}_{n}\right)$. Hence, these higher order terms converge to 0 as $m, n \rightarrow+\infty$ in the $C^{r}$ topology.

It remains to check the convergence of $(m, n)$-dependent terms involving $\tilde{H}_{1}$ and $\tilde{H}_{2}$ in the expression of $\bar{z}_{1}$. These estimations follow from 5.12 . Thus, we finish checking the convergence for higher order terms containing $\tilde{H}_{1}, \tilde{H}_{2}$ and $\tilde{H}_{3}$.

- Higher order terms containing $H_{1}, H_{2}, H_{3}$. We start by evaluating the expressions of $\bar{x}_{1}$ and $\bar{z}_{1}$. From (3.5), $H_{1}$ and $H_{3}$ start from degree two terms. Hence, the orders of $H_{1}$ and $H_{3}$ are dominated by those of $x_{m}^{2}, y_{m}^{2}, z_{m}^{2}, x_{m} y_{m}, y_{m} z_{m}, z_{m} x_{m}$, which can be evaluated directly from (5.8). In this way, one can check the terms containing $H_{1}$ and $H_{3}$ in the expressions of $\bar{x}_{1}$ and $\bar{z}_{1}$ in (5.9) and (5.11) as follows: for $i=1,3$,

$$
\sigma^{n} \tilde{\sigma}^{m} H_{i}\left(\mathbf{x}_{m}\right)=O\left(\tilde{\lambda}^{2 m} \sigma^{n} \tilde{\sigma}^{m}\right)+O\left(\sigma^{-3 n} \tilde{\sigma}^{-5 m}\right)+O\left(\sigma^{n} \tilde{\sigma}^{3 m} \zeta^{4 n}\right)+O\left(\sigma^{-n} \tilde{\sigma}^{-m}\right) .
$$

This converges to 0 under the conditions 1.3)-1.4 as $m, n \rightarrow \infty$. Moreover, this and (5.8) imply that the $r$ th order derivatives of $\sigma^{n} \tilde{\sigma}^{m} H_{i}\left(\mathbf{x}_{m}\right)$ also converge to 0 as $m, n \rightarrow+\infty$.

Next, we evaluate the expressions of $\bar{y}_{1}$ which only contains terms $H_{2}$. From (3.5), $H_{2}\left(\mathbf{x}_{m}\right)$ starts from the terms of $x_{m}^{2}, x_{m} y_{m}$ and $z_{m} x_{m}$. From 5.8 with (1.4), we only have to check the order of terms for $x_{m} y_{m}$ and $z_{m} x_{m}$. Note that from (5.8),

$$
\sigma^{2 n} \tilde{\sigma}^{2 m} x_{m} y_{m}=O\left(\tilde{\lambda}^{m} \tilde{\sigma}^{-m}\right)+O\left(\tilde{\lambda}^{m} \sigma^{2 n} \tilde{\sigma}^{3 m} \zeta^{2 n}\right), \sigma^{2 n} \tilde{\sigma}^{2 m} z_{m} x_{m}=O\left(\tilde{\lambda}^{m} \sigma^{n} \tilde{\sigma}^{m}\right),
$$

both of which converge to 0 under $(1.3)-1.4$ as $m, n \rightarrow \infty$. This implies that $\sigma^{2 n} \tilde{\sigma}^{2 m} H_{2}$ and its $r$ th order derivatives tend to 0 as $m, n \rightarrow+\infty$.

- Conclusion. Recall that $\lambda^{n} \tilde{\zeta}^{m}$ converges to $\left(\gamma_{1} a_{3}\right)^{-1} \xi$. Hence, due to the above evaluations, 5.9 -5.11) converge to the following

$$
(\bar{x}, \bar{y}, \bar{z}) \longmapsto\left(\xi \bar{x}+a_{2} \beta_{2} \bar{y}, \bar{\mu}+b_{3}\left(\xi a_{3}^{-1}\right)^{2} \bar{x}^{2}+\beta_{2}^{2} b_{2} \bar{y}^{2}+\xi a_{3}^{-1} \beta_{2} b_{4} \bar{x} \bar{y}, c_{2} \beta_{2} \bar{y}\right)
$$

in the $C^{r}$ topology on compact domains.

Finally, to improve the appearance of (5.14), consider the next coordinate change

$$
\Theta:(\bar{x}, \bar{y}, \bar{z}, \bar{\mu}) \longmapsto\left(\beta_{2} a_{2}^{-1} b_{2} \bar{x}, \beta_{2}^{2} b_{2} \bar{y}, \beta_{2} c_{2}^{-1} b_{2} \bar{z}, \beta_{2}^{2} b_{2} \bar{\mu}\right)=:(\tilde{x}, \tilde{y}, \tilde{z}, \tilde{\mu}) .
$$

By $\Theta,(5.14)$ is conjugate to

$$
(\tilde{x}, \tilde{y}, \tilde{z}) \longmapsto\left(\xi \tilde{x}+\tilde{y}, \tilde{\mu}+\tilde{y}^{2}+\kappa_{1} \tilde{x}^{2}+\kappa_{2} \tilde{x} \tilde{y}, \tilde{y}\right),
$$

where (remembering the condition (3.7))

$$
\kappa_{1}=\left(\xi a_{2} a_{3}^{-1}\right)^{2} b_{2}^{-1} b_{3}, \quad \kappa_{2}=\xi a_{2}\left(a_{3} b_{2}\right)^{-1} b_{4} .
$$

This ends the proof of Theorem 1.2 . (3).

\section{Robust COnNECTIONS BETWEen SADDLES OF DIFFERENT INDICES}

In this section, we prove Theorem 1.4 , that is, we give the perturbation to obtain the connection between the blender and the saddle $Q$. 
6.1. Further perturbation. Let $\left\{f_{\mu, \nu}\right\}=\left\{f_{\mu_{k}(\bar{\mu}), \nu_{k}}\right\}$ be the family in Theorem 1.2. We consider another additional $n$-dependent perturbation $\left\{g_{n}\right\}=\left\{g_{n_{k}}\right\}$ of $\left\{f_{\mu, \nu}\right\}$ in a small neighborhood of $X=(0,1,0)$ in $U_{Q}$ to show Theorem 1.4. In the following, to simplify the notations, we again drop the subscript $k$ of $m_{k}$ and $n_{k}$.

Let us consider a smooth bump function $b$ satisfying the following:

- $b(t)=0$ for $|t|>1 / 2$.

- $b(t)=1$ for $|t|<1 / 3$.

- $0 \leq b(t) \leq 1$.

Then, given $n>0$, consider the functions $B, B_{n}: \mathbb{R}^{3} \rightarrow \mathbb{R}$ defined as follows:

- $B(x, y, z):=b(x) b(y) b(z)$,

- $B_{n}(x, y, z):=\lambda^{n} B\left(x / \zeta^{n}, y / \zeta^{n}, z / \zeta^{n}\right)$,

and an $n$-dependent neighborhood of $X=(0,1,0)$ defined as follows:

$$
U_{X, n}=U_{X}=\left\{(x, 1+y, z)|| x|,| y|,| z \mid<\zeta^{n} / 2 .\right\} .
$$

Note that $U_{X}$ converges to $\{X\}$ as $n \rightarrow+\infty$. We define $\theta_{n}: M \rightarrow M$ as follows:

- if $(x, 1+y, z) \in U_{X}$,

$$
\theta_{n}(x, 1+y, z):=(x, 1+y, z)+\left(B_{n}(x, y, z), 0,0\right) ;
$$

- otherwise, $\theta_{n}$ is the identity map.

Finally, we define

$$
g_{n}:=\theta_{n} \circ f_{\mu_{k}(\bar{\mu}), \nu_{k}} .
$$

For this perturbation, we prove the following:

Lemma 6.1. Suppose $\lambda / \zeta^{1+\alpha}<1$. Then the $C^{1+\alpha}$ distance between $g_{n_{k}}$ and $f_{\mu, \nu}=f_{\mu_{k}(\bar{\mu}), \nu_{k}}$ goes to zero as $k$ (hence $n_{k}$ ) tends to $+\infty$.

Note that the condition $\lambda / \zeta^{1+\alpha}<1$ is equivalent to $\alpha<\frac{\log \lambda}{\log \zeta}-1$.

Proof. The convergence in the $C^{0}$ topology is easy to see. To see the $C^{1+\alpha}$ convergence, we only need to check the $C^{1+\alpha}$ smallness of each partial derivative of $B_{n}$ for large $n$. By the symmetry, we only confirm for $\left(B_{n}\right)_{x}$ (partial derivative of $B_{n}$ with respect to $x)$ and omit the check for $\left(B_{n}\right)_{y}$ and $\left(B_{n}\right)_{z}$.

The partial derivative $\left(B_{n}\right)_{x}$ is given as follows:

$$
\left(B_{n}\right)_{x}=\left(B_{n}\right)_{x}(x, y, z):=\left(\frac{\lambda}{\zeta}\right)^{n} b^{\prime}\left(\frac{x}{\zeta^{n}}\right) b\left(\frac{y}{\zeta^{n}}\right) b\left(\frac{z}{\zeta^{n}}\right) .
$$

Then, since $\lambda / \zeta<\lambda / \zeta^{1+\alpha}<1$, we can see the the $C^{1}$ smallness of this function for large $n$.

Let us confirm the smallness of $\alpha$-Hölder constant for large $n$. Given $\left(x_{0}, y_{0}, z_{0}\right)$, $(x, y, z) \in \mathbb{R}^{3}$, we have

$$
\begin{aligned}
& \frac{\left(B_{n}\right)_{x}\left(x_{0}+x, y_{0}+y, z_{0}+z\right)-\left(B_{n}\right)_{x}\left(x_{0}, y_{0}, z_{0}\right)}{|(x, y, z)|^{\alpha}} \\
= & \left(\frac{\lambda}{\zeta^{1+\alpha}}\right)^{n} \frac{b^{\prime}\left(\frac{x_{0}+x}{\zeta^{n}}\right) b\left(\frac{y_{0}+y}{\zeta^{n}}\right) b\left(\frac{z_{0}+z}{\zeta^{n}}\right)-b^{\prime}\left(\frac{x_{0}}{\zeta^{n}}\right) b\left(\frac{y_{0}}{\zeta^{n}}\right) b\left(\frac{z_{0}}{\zeta^{n}}\right)}{|(x / \zeta, y / \zeta, z / \zeta)|^{\alpha}} .
\end{aligned}
$$

In the last formula, $\left(\lambda / \zeta^{1+\alpha}\right)^{n}$ converges to zero as $n \rightarrow+\infty$ if $\left(\lambda / \zeta^{1+\alpha}\right)<1$, and the absolute value of the rest of the formula is bounded by the Hölder constant 
of $b^{\prime}(x) b(y) b(z)$, which does not depend on $n$ and $\left(x_{0}, y_{0}, z_{0}\right)$. This shows that the $\alpha$-Hölder constant of $\left|\left(B_{n}\right)_{x}\right|$ converges to 0 as $n$ tends to $+\infty$.

This perturbation may give some effect on points in the renormalizations. However, we can prove the following:

Lemma 6.2. The perturbation $\theta_{n}$ does not give any effect on the first return map of the renormalization. More precisely, for every $X \in \Phi_{m, n}(\Delta)$, we have $\left(g_{n}\right)^{i}(X)=$ $\left(f_{\mu, \nu}\right)^{i}(X)$ for $i \in\left[0, N_{2}+m+N_{1}+n\right]$.

In particular, the blender of $f_{\mu, \nu}$ is not affected by the perturbation. Thus $g_{n}$ also has the same blender.

Proof. Take $X \in \Phi_{m, n}(\Delta)$. The only possibility where $\left(g_{n}\right)^{i}(X)$ may get some effect from the perturbation is that $\left(g_{n}\right)^{n}(X)$ is contained in the support of the perturbation $U_{X}$. Thus, let us calculate the position of $\left(g_{n}\right)^{n}(X)$.

A point $X \in \Phi_{m, n}(\Delta)$ has the following form:

$$
\left(\sigma^{-n} \tilde{\sigma}^{-m} x+1, \sigma^{-2 n} \tilde{\sigma}^{-2 m} y+\sigma^{-n}, \sigma^{-n} \tilde{\sigma}^{-m} z+1\right),
$$

where the point $(x, y, z)$ is chosen from some compact domain. Hence, the coordinate of $\left(f_{\mu, \nu}\right)^{n}(X)$ is given as

$$
\left(\lambda^{n} \sigma^{-n} \tilde{\sigma}^{-m} x+\lambda^{n}, \sigma^{-n} \tilde{\sigma}^{-2 m} y+1, \zeta^{n} \sigma^{-n} \tilde{\sigma}^{-m} z+\zeta^{n}\right) .
$$

We show that, if $n$ is sufficiently large, then the $z$-coordinate of this point is greater than $\zeta^{n} / 2$, which is the $z$-coordinate of the boundary of $U_{X}$. This implies that the point $\left(g_{n}\right)^{n}(X)$ is outside the support of the perturbation.

To see this, we take the quotient of these two quantities:

$$
\frac{\zeta^{n} / 2}{\zeta^{n} \sigma^{-n} \tilde{\sigma}^{-m} z+\zeta^{n}}=\frac{1}{2\left(\sigma^{-n} \tilde{\sigma}^{-m} z+1\right)} .
$$

If $z$ is bounded, then by taking sufficiently large $m$ and $n$, one can check that the last term lies in the interval $(0,1)$, which completes the proof.

6.2. Proof of Theorem 1.4. The perturbation above keeps $Q_{g}=Q$ intact. Meanwhile, the local unstable manifold of $Q$ is no longer a straight line in $U_{Q}$. Indeed, $W_{\text {loc }}^{u}(Q)$ has a bumped sub-arc near $X$ which contains the vertical part given as

$$
\ell=\ell_{m, n}:=\left\{\left(\lambda^{n}, 1+\sigma^{-n} \tilde{\sigma}^{-2 m} y, 0\right)|| y \mid<4 \beta_{2}^{2} b_{2}\right\}
$$

see Figure 6.1. Let us consider another segment

$$
\hat{\ell}=\hat{\ell}_{m, n}:=\left\{\Phi_{m, n}(0, y, 0)|| y \mid<4\right\},
$$

which is contained in the boundary of the box

$$
\Delta_{m, n}:=\Phi_{m, n}(\Delta),
$$

where $\Phi_{m, n}:=\Psi_{m, n} \circ \Theta \circ \tilde{\Theta}$, see $5.2, \sqrt{5.15}$ and $\sqrt{2.2}$ for definitions and $\Delta$ is the box given in 2.4 . In the next proposition, we compare the $g^{N_{2}+m+N_{1}}$-images of $\ell$ and $\hat{\ell}$.

Proposition 6.3. The $C^{1}$ distance between the $\left(g^{N_{2}+m+N_{1}}\right)$-images of $\ell$ and that of $g^{n}(\hat{\ell})$, measured in the $\Phi_{m, n}$-coordinate, becomes arbitrarily small by letting $k$ (hence $(m, n)$ ) large (remember that $k$ is the subscript for renormalizations in Theorem 1.2). 


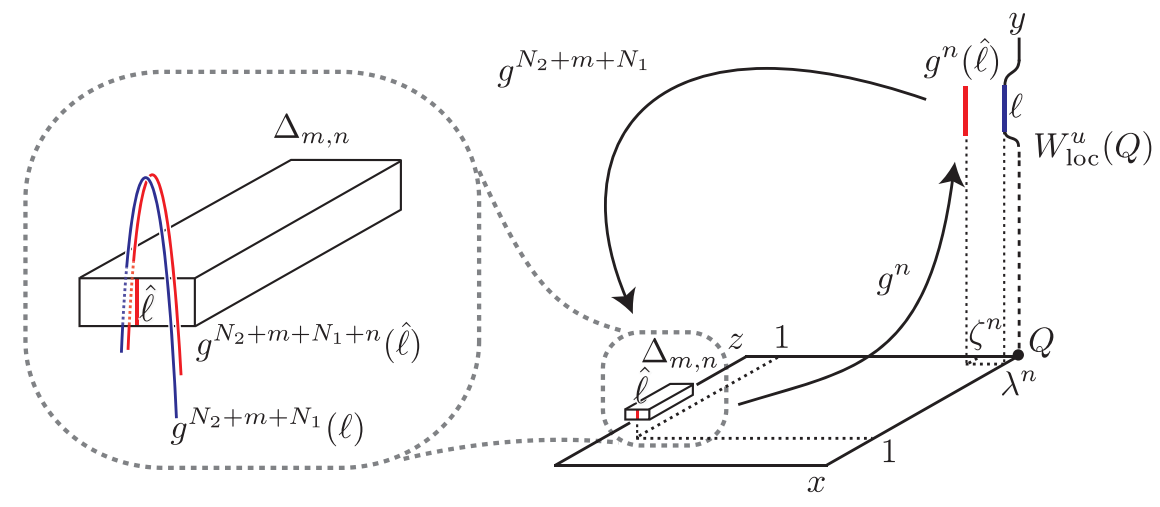

Figure 6.1.

By Remark 2.6, $g_{N_{2}+m+N_{1}+n}(\hat{\ell})$ has non-empty intersection with the stable manifold of the blender $\Lambda_{g}$. Hence, Proposition 6.3 implies that in the perturbed system the unstable manifold $W^{u}(Q)$ has non-empty intersection with the superposition region of the blender $\Lambda_{g}$ as a vertical segment for sufficiently large $m, n$. This concludes Theorem 1.4 .

Thus, we only need to prove Proposition 6.3. Let us start the proof.

Proof. The proof is obtained by similar calculations in Theorem 1.2. (2). In the following, we give explicit calculations of image of two segments with respect to $\Psi_{m, n}$-coordinate and prove the smallness of the $C^{1}$ distance of these two segments. Since the coordinate change $\Theta \circ \tilde{\Theta}$ is bounded (independent of $m$ and $n$ ), it implies the $C^{1}$ smallness of the difference of two segments in the $\Phi_{m, n}$-coordinate.

- Calculation of $\hat{\ell}$. First, for any $(0, t, 0) \in \Phi_{m, n}^{-1}(\hat{\ell})$ where $|t|<4$, we put

$$
(\hat{x}(t), \hat{y}(t), \hat{z}(t)):=\Psi_{m, n}^{-1} \circ g^{N_{2}+m+N_{1}+n} \circ \Phi_{m, n}(0, t, 0) .
$$

Note that by Lemma 6.2 , we know that $\hat{\ell}$ does not get any effect by the perturbation. Thus by the same procedure as in (5.9)-(5.11), each entry of $(\hat{x}(t), \hat{y}(t), \hat{z}(t))$ is given as follows:

$$
\begin{aligned}
\hat{x}(t)=\left(\tilde{\lambda}^{m} \tilde{\sigma}^{-m} a_{1} \alpha_{2}+a_{2} \beta_{2}\right) t+\sigma^{n} \tilde{\lambda}^{m} \tilde{\sigma}^{m} a_{1} \tilde{H}_{1}\left(\hat{\tilde{\mathbf{x}}}_{n}(t)\right)+\sigma^{n} \tilde{\sigma}^{2 m} a_{2} \tilde{H}_{2}\left(\hat{\tilde{\mathbf{x}}}_{n}(t)\right) \\
\quad+\sigma^{n} \tilde{\zeta}^{m} \tilde{\sigma}^{m} a_{3} \tilde{H}_{3}\left(\hat{\mathbf{x}}_{n}(t)\right)+\sigma^{n} \tilde{\sigma}^{m} H_{1}\left(\hat{\mathbf{x}}_{m}(t)\right) ; \\
\hat{y}(t)= \\
\quad \bar{\mu}+\sigma^{n} \tilde{\lambda}^{m} b_{1} \alpha_{2} t+\beta_{2}^{2} b_{2} t^{2}+\sigma^{2 n} \tilde{\lambda}^{m} \tilde{\sigma}^{2 m} b_{1} \tilde{H}_{1}\left(\hat{\tilde{\mathbf{x}}}_{n}(t)\right)+2 t \sigma^{n} \tilde{\sigma}^{2 m} \beta_{2} b_{2} \tilde{H}_{2}\left(\hat{\tilde{\mathbf{x}}}_{n}(t)\right) \\
+t \sigma^{n} \tilde{\zeta}^{m} \tilde{\sigma}^{m} \beta_{2} b_{4} \tilde{H}_{3}\left(\hat{\tilde{\mathbf{x}}}_{n}(t)\right)+\sigma^{2 n} \tilde{\zeta}^{m} \tilde{\sigma}^{3 m} b_{4} \tilde{H}_{2}\left(\hat{\tilde{\mathbf{x}}}_{n}(t)\right) \tilde{H}_{3}\left(\hat{\tilde{\mathbf{x}}}_{n}(t)\right) \\
\quad+\sigma^{2 n} \tilde{\sigma}^{4 m} b_{2} \tilde{H}_{2}\left(\tilde{\tilde{\mathbf{x}}}_{n}(t)\right)^{2}+\sigma^{2 n} \tilde{\zeta}^{2 m} \tilde{\sigma}^{2 m} b_{3} \tilde{H}_{3}\left(\hat{\tilde{\mathbf{x}}}_{n}(t)\right)^{2}+\sigma^{2 n} \tilde{\sigma}^{2 m} H_{2}\left(\hat{\mathbf{x}}_{m}(t)\right) ; \\
\hat{z}(t)=\left(\tilde{\lambda}^{m} \tilde{\sigma}^{-m} c_{1} \alpha_{2}+c_{2} \beta_{2}\right) t+\sigma^{n} \tilde{\lambda}^{m} \tilde{\sigma}^{m} c_{1} \tilde{H}_{1}\left(\hat{\tilde{\mathbf{x}}}_{n}(t)\right) \\
+\sigma^{n} \tilde{\sigma}^{2 m} c_{2} \tilde{H}_{2}\left(\hat{\tilde{\mathbf{x}}}_{n}(t)\right)+\sigma^{n} \tilde{\sigma}^{m} H_{3}\left(\hat{\mathbf{x}}_{m}(t)\right) .
\end{aligned}
$$

Here, $\hat{\tilde{\mathbf{x}}}_{n}(t)$ and $\hat{\mathbf{x}}_{m}(t)$ in the higher order terms are given as

$$
\hat{\tilde{\mathbf{x}}}_{n}(t)=g^{n} \circ \Phi_{m, n}(0, t, 0)-(0,1,0), \hat{\mathbf{x}}_{m}(t)=g^{m+N_{1}+n} \circ \Phi_{m, n}(0, t, 0)-(0,1,1) .
$$


- Calculation of $\ell$. Next, for any point $\left(\lambda^{n}, 1+\sigma^{-n} \tilde{\sigma}^{-2 m} t, 0\right) \in \ell$ where $|t|<$ $4 \beta_{2}^{2} b_{2}$, let us calculate its image under $\Psi_{m, n}^{-1} \circ g^{N_{2}+m+N_{1}}$. First, if $m, n$ are sufficiently large, then $\ell$ is contained in the domain of the definition of the transition from $U_{X}$ to $U_{Y}$. Thus $g^{N_{1}}(\ell) \subset U_{P}$. Then, we calculate $g^{m+N_{1}}(\ell):=$ $(\mathrm{x}(t), \mathrm{y}(t)+1, \mathrm{z}(t)+1)$. They are given as follows:

$$
\begin{aligned}
& \mathbf{x}(t)=\tilde{\lambda}^{m}+t \sigma^{-n} \tilde{\sigma}^{-2 m} \alpha_{2}-\zeta^{n} \tilde{\lambda}^{m} \alpha_{3}+\tilde{\lambda}^{m} \tilde{H}_{1}\left(\tilde{\mathbf{x}}_{n}(t)\right), \\
& \mathrm{y}(t)=t \sigma^{-n} \tilde{\sigma}^{-m} \beta_{2}+\tilde{\sigma}^{m} \tilde{H}_{2}\left(\tilde{\mathbf{x}}_{n}(t)\right), \\
& \mathbf{z}(t)=\tilde{\zeta}^{m} \tilde{H}_{3}\left(\tilde{\mathbf{x}}_{n}(t)\right) .
\end{aligned}
$$

By a similar argument to obtain (5.8), we can check that these three numbers converge to zero as $m, n$ tends to $+\infty$. Hence these points are in the domain of transition map from $U_{P}$ to $U_{Q}$, see also Remark 6.4 after the end of this proof.

Finally, we calculate

$$
(x(t), y(t), z(t)):=\Psi_{m, n}^{-1} \circ g^{N_{2}+m+N_{1}}\left(\lambda^{n}, 1+\sigma^{-n} \tilde{\sigma}^{-2 m} t, 0\right) .
$$

By the same computation as the proof of Theorem 1.2 (2), one has

$$
\begin{gathered}
x(t)=\left(\tilde{\lambda}^{m} \tilde{\sigma}^{-m} a_{1} \alpha_{2}+a_{2} \beta_{2}\right) t+\sigma^{n} \tilde{\lambda}^{m} \tilde{\sigma}^{m} a_{1} \tilde{H}_{1}\left(\tilde{\mathbf{x}}_{n}(t)\right)+\sigma^{n} \tilde{\sigma}^{2 m} a_{2} \tilde{H}_{2}\left(\tilde{\mathbf{x}}_{n}(t)\right) \\
+\sigma^{n} \tilde{\zeta}^{m} \tilde{\sigma}^{m} a_{3} \tilde{H}_{3}\left(\tilde{\mathbf{x}}_{n}(t)\right)+\sigma^{n} \tilde{\sigma}^{m} H_{1}\left(\tilde{\mathbf{x}}_{m}(t)\right)-\tilde{\lambda}^{m} \zeta^{n} \tilde{\sigma}^{m} \sigma^{n} a_{1} \alpha_{3} ; \\
\begin{array}{r}
y(t)=\bar{\mu}+\sigma^{n} \tilde{\lambda}^{m} b_{1} \alpha_{2} t+\beta_{2}^{2} b_{2} t^{2}+\sigma^{2 n} \tilde{\lambda}^{m} \tilde{\sigma}^{2 m} b_{1} \tilde{H}_{1}\left(\tilde{\mathbf{x}}_{n}(t)\right)+2 t \sigma^{n} \tilde{\sigma}^{2 m} \beta_{2} b_{2} \tilde{H}_{2}\left(\tilde{\mathbf{x}}_{n}(t)\right) \\
+t \sigma^{n} \tilde{\zeta}^{m} \tilde{\sigma}^{m} \beta_{2} b_{4} \tilde{H}_{3}\left(\tilde{\mathbf{x}}_{n}(t)\right)+\sigma^{2 n} \tilde{\zeta}^{m} \tilde{\sigma}^{3 m} b_{4} \tilde{H}_{2}\left(\tilde{\mathbf{x}}_{n}(t)\right) \tilde{H}_{3}\left(\tilde{\mathbf{x}}_{n}(t)\right) \\
+\sigma^{2 n} \tilde{\sigma}^{4 m} b_{2} \tilde{H}_{2}\left(\tilde{\mathbf{x}}_{n}(t)\right)^{2}+\sigma^{2 n} \tilde{\zeta}^{2 m} \tilde{\sigma}^{2 m} b_{3} \tilde{H}_{3}\left(\tilde{\mathbf{x}}_{n}(t)\right)^{2} \\
\quad+\sigma^{2 n} \tilde{\sigma}^{2 m} H_{2}\left(\tilde{\mathbf{x}}_{m}(t)\right)-\tilde{\lambda}^{m} \zeta^{n} \tilde{\sigma}^{2 m} \sigma^{2 n} b_{1} \alpha_{3} ; \\
z(t)=\left(\tilde{\lambda}^{m} \tilde{\sigma}^{-m} c_{1} \alpha_{2}+c_{2} \beta_{2}\right) t+\sigma^{n} \tilde{\lambda}^{m} \tilde{\sigma}^{m} c_{1} \tilde{H}_{1}\left(\tilde{\mathbf{x}}_{n}(t)\right) \\
+\sigma^{n} \tilde{\sigma}^{2 m} c_{2} \tilde{H}_{2}\left(\tilde{\mathbf{x}}_{n}(t)\right)+\sigma^{n} \tilde{\sigma}^{m} H_{3}\left(\tilde{\mathbf{x}}_{m}(t)\right)-\tilde{\lambda}^{m} \zeta^{n} \tilde{\sigma}^{m} \sigma^{n} c_{1} \alpha_{3},
\end{array}
\end{gathered}
$$

where

$$
\tilde{\mathbf{x}}_{n}(t)=\left(\lambda^{n}, \sigma^{-n} \tilde{\sigma}^{-2 m} t, 0\right), \tilde{\mathbf{x}}_{m}(t)=g^{m+N_{1}}\left(\lambda^{n}, 1+\sigma^{-n} \tilde{\sigma}^{-2 m} t, 0\right)-(0,1,1) .
$$

Note that convergence of the higher order terms in the above $(\tilde{x}(t), \tilde{y}(t), \tilde{z}(t))$ and $(x(t), y(t), z(t))$ are already contained in the proof of Theorem $1.2+(2)$. Then we have

$$
\|(x(t), y(t), z(t))-(\tilde{x}(t), \tilde{y}(t), \tilde{z}(t))\|_{C^{1}} \leqslant O\left(\tilde{\lambda}^{m} \zeta^{n} \tilde{\sigma}^{2 m} \sigma^{2 n}\right) .
$$

The conditions 1.3-1.4 imply that $\tilde{\lambda}^{m} \zeta^{n} \tilde{\sigma}^{2 m} \sigma^{2 n}$ converges to 0 as $m, n \rightarrow \infty$.

Remark 6.4. Let us discuss the importance of the perturbation $\theta_{n}$. If we do not give the perturbation, then $g^{m+N_{1}}(\ell)$ is in general not contained in the domain of the transition $f^{N_{2}}$. More precisely, without $\theta_{n}$, in the $y$-coordinate of $g^{m+N_{1}}(\ell)$ there remains a term of the form $\lambda^{n} \tilde{\zeta}^{m} \beta_{2}$ which converges to some non-zero constant. The perturbation $\theta_{n}$ is performed so as to annihilate this term.

Acknowledgements. The authors thank Hiroshi Kokubu and Ming-Chia Li for financial support which brought us together. We also thank the warm hospitality of Kyoto University, NCTU, and PUC-Rio. This paper was partially supported by GCOE (Kyoto University), JSPS KAKENHI Grant Number 22540226, CNPq (Research and PDJ grants), Faperj (CNE), Palis-Balzan project and the Aihara Project, the FIRST program from JSPS, initiated by CSTP. 


\section{REFERENCES}

[1] P. Barrientos, Y. Ki and A. Raibekas, Symbolic blender-horseshoes and applications arXiv: 1211.7088

[2] Ch. Bonatti and L. J. Díaz, Persistent nonhyperbolic transitive diffeomorphisms, Ann. Math. (2) 143 (1996), no. 2, 357-396.

[3] Ch. Bonatti and L. J. Díaz On maximal transitive sets of generic diffeomorphisms, Publ. Math. I.H.É.S. 96 (2003) 171-197.

[4] Ch. Bonatti and L. J. Díaz Robust heterodimensional cycles and $C^{1}$-generic dynamics, Journal of the Inst. of Math. Jussieu, 7(3) (2008), 469-525.

[5] Ch. Bonatti, L. J. Díaz, Abundance of $C^{1}$-robust homoclinic tangencies, Trans. Amer. Math. Soc. 364 (2012), 5111-5148.

[6] Ch. Bonatti, L. J. Díaz and M. Viana, Dynamics beyond uniform hyperbolicity, Encyclopedia of Mathematical Sciences (Mathematical Physics) 102, Mathematical physics, III. Springer Verlag, 2005.

[7] L. J. Díaz, Robust nonhyperbolic dynamics and heterodimensional cycles, Ergod. Theory Dynam. Syst., 15 (1995), 291-315.

[8] L. J. Díaz, A. Nogueira and E. R. Pujals, Heterodimensional tangencies, Nonlinearity, 19 (2006), 2543-2566.

[9] S. Gonchenko and M.-Ch. Li, Shilnikov's cross-map method and hyperbolic dynamics of three-dimensional Hénon-like maps, Regul. Chaotic Dyn., 15-2 (2010), 165-184.

[10] S. Hayashi, Connecting invariant manifolds and the solution of the $C^{1}$ stability and $\Omega$ stability conjectures for flows, Ann. of Math. (2) 145 (1997), 81-137.

[11] S. Kiriki, Y. Nishizawa and T. Soma, Heterodimensional tangencies on cycles leading to strange attractors, Discrete Conti. Dynam. Sys., 27-1 (2010), 285-300.

[12] S. Kiriki and T. Soma, $C^{2}$-robust heterodimensional tangencies, Nonlinearity, 25 (2012), 3277-3299.

[13] M. Nassiri and E. R. Pujals, Robust transitivity in Hamiltonian dynamics, Ann. Sci. Éc. Norm. Supér., 45 (2012), 191-239.

[14] S. E. Newhouse, Nondensity of axiom A(a) on $S^{2}$, Global Analysis (Proc. Sympos. Pure Math., Vol. XIV, Berkeley, Calif., Amer. Math. Soc. (1968) 191-202.

[15] S. E. Newhouse, The abundance of wild hyperbolic sets and non-smooth stable sets for diffeomorphisms, Publ. Math. I.H.É.S., 50 (1979), 101-151.

[16] J. Palis and F. Takens, Hyperbolicity and sensitive chaotic dynamics at homoclinic bifurcations, Fractal dimensions and infinitely many attractors, Cambridge Studies in Advanced Mathematics 35, Cambridge University Press, Cambridge, 1993.

[17] J. Palis and M. Viana, High dimension diffeomorphisms displaying infinitely many periodic attractors, Ann. of Math. (2), 140 (1994), 207-250.

[18] N. Romero, Persistence of homoclinic tangencies in higher dimensions, Ergod. Th. Dynam. Sys., 15 (1995), no. 4, 735-757.

[19] S. Sternberg, On the structure of local homeomorphisms of euclidean $n$-space, II, Amer. J. Math., 80 (1958), 623-631.

[20] F. Takens, Partially hyperbolic fixed points, Topology 10 (1971), 133-147.

Depto. Matemática, PUC-Rio, Marquês de S. Vicente 225 22453-900 Rio de Janeiro RJ BRAZIL

E-mail address: lodiaz@mat.puc-rio.br

Dept. of Mathematics, Tokai University, 4-1-1 Kitakaname, Hiratuka Kanagawa, 259-1292, JAPAN

E-mail address: kiriki@tokai-u.jp

First, Ainara Innovative Mathematical Modelling Project, Institute of Industrial Science, The University of Tokyo, 4-6-1 Komaba, Meguro-Ku, Tokyo 153-8505, Japan

E-mail address: herrsinon@07.alumni.u-tokyo.ac.jp 